\title{
CRISPR/Cas9-mediated targeted chromosome elimination
}

\author{
Erwei Zuo ${ }^{1 \dagger}$, Xiaona Huo ${ }^{1 \dagger}$, Xuan Yao ${ }^{1 \dagger}$, Xinde Hu${ }^{1 \dagger}$, Yidi Sun ${ }^{3 \dagger}$, Jianhang Yin ${ }^{2 \dagger}$, Bingbing He $\mathrm{H}^{1,4}$, Xing Wang ${ }^{1,4}$, \\ Linyu Shi ${ }^{1}$, Jie Ping ${ }^{5}$, Yu Wei ${ }^{1,6}$, Wenqin Ying ${ }^{1}$, Wei Wei ${ }^{1,7}$, Wenjia Liu ${ }^{1}$, Cheng Tang ${ }^{1}$, Yixue Li ${ }^{3}$, Jiazhi Hu ${ }^{2^{*}}$ and \\ Hui Yang ${ }^{1 *}$
}

\begin{abstract}
Background: The CRISPR/Cas9 system has become an efficient gene editing method for generating cells carrying precise gene mutations, including the rearrangement and deletion of chromosomal segments. However, whether an entire chromosome could be eliminated by this technology is still unknown.

Results: Here we demonstrate the use of the CRISPR/Cas9 system to eliminate targeted chromosomes. Using either multiple cleavages induced by a single-guide RNA (sgRNA) that targets multiple chromosome-specific sites or a cocktail of multiple sgRNAs, each targeting one specific site, we found that a sex chromosome could be selectively eliminated in cultured cells, embryos, and tissues in vivo. Furthermore, this approach was able to produce a targeted autosome loss in aneuploid mouse embryonic stem cells with an extra human chromosome and human induced pluripotent stem cells with trisomy 21, as well as cancer cells.
\end{abstract}

Conclusions: CRISPR/Cas9-mediated targeted chromosome elimination offers a new approach to develop animal models with chromosome deletions, and a potential therapeutic strategy for human aneuploidy diseases involving additional chromosomes.

\section{Background}

Aneuploidy is a human genetic disorder due to the addition or deletion of a chromosome, leading to significant morbidity and mortality during infancy or childhood [1]. The past decade has witnessed major advances in strategies to correct single-gene defects of rare monogenic disorders, beginning with in vitro experiments and in several cases advancing to in vivo studies and clinical trials. By contrast, only a few attempts have been made to genetically correct the over-dose of genes for an entire chromosome in aneuploid cells. Targeted chromosome elimination could be achieved by insertion of oppositely oriented loxP sites into the targeted chromosome followed by Cre-mediated sister-

\footnotetext{
*Correspondence: hujz@pku.edu.cn; huiyang@ion.ac.cn

${ }^{\dagger}$ Equal contributors

${ }^{2}$ State Key Laboratory of Membrane Biology and Minstry of Education Key Laboratory of Cell Proliferation and Differentiation, School of Life Sciences, Peking-Tsinghua Center for Life Sciences, Peking University, Beijing 100871, China

${ }^{1}$ Institute of Neuroscience, State Key Laboratory of Neuroscience, Key Laboratory of Primate Neurobiology, CAS Center for Excellence in Brain Science and Intelligence Technology, Shanghai Institutes for Biological Sciences, Chinese Academy of Sciences, Shanghai 200031, China Full list of author information is available at the end of the article
}

chromatid recombination [2], or by insertion of a TKNEO transgene into one copy of a targeted chromosome followed by drug selection of chromosome-deletion clones via spontaneous chromosome loss [3]. Both of these approaches require two-step manipulation and resulted in low yields of chromosome-deleted cells, and are thus unsuitable for in vivo studies. Alternatively, over-dose of genes in aneuploid cells could be corrected by insertion of a large, inducible XIST transgene into the targeted chromosome to silence one copy of it [4]. However, the efficiency of the targeted insertion was very low and some genes may have escaped from inactivation.

The type II bacterial CRISPR/Cas9 system has been engineered into an efficient genome-editing tool consisting of the Cas9 nuclease and a single guide RNA (sgRNA), dramatically transforming our ability to edit the genomes of diverse organisms. The sgRNA targets Cas9 to genomic regions to induce double-stranded DNA breaks, which are repaired by nonhomologous end-joining or homology-directed repair. CRISPR/Cas9mediated genome editing has been applied to generate cells or animals carrying precise gene mutations $[5,6]$, 
including rearrangements $[7,8]$ and deletion of chromosome segments [9]. We asked whether this powerful technology could be used for targeted chromosome elimination to generate animal models with chromosome deletion in various species and to treat human aneuploidy diseases involving chromosome addition.

In this study we report a novel application of CRISPR/ Cas9 technology; the selective elimination of a single specific chromosome via multiple DNA cleavages on the targeted chromosome in cultured cells, embryos, and in vivo tissues. These cleavages were induced by a single sgRNA or two sgRNAs that targeted multiple chromosome-specific sites, or by a cocktail of 14 sgRNAs, with each targeting one specific site. More importantly, this approach eliminated human chromosome 21 (hChr21) in human induced pluripotent stem cells (iPSCs) with trisomy 21. CRISPR/ Cas9-mediated targeted chromosome elimination offers a new approach to developing animal models and therapeutic treatments for aneuploidy.

\section{Results}

\section{Elimination of the $\mathrm{Y}$ chromosome in vitro and in vivo}

We initially examined whether complete elimination of a chromosome could be achieved efficiently by using CRISPR/Cas9-mediated multiple cuts at chromosomespecific sites. First, we examined whether the mouse Y chromosome contains unique repeated sequences that could be used for large-scale chromosomal editing via short-guide RNAs (sgRNAs), and whether such editing could result in $\mathrm{Y}$ chromosome deletion. Sequence analysis for all mouse chromosomes, using 23-bp sgRNA target sequences containing an adjacent 'NGG' protospacer adjacent motif (PAM), showed that each chromosome indeed has unique and multiple repeated sequences for targeting by a single specific sgRNA (Additional file 1: Table S1 and Additional file 2: Table S2). These repeated sequences appeared either clustered at one region or scattered across the entire chromosome (Fig. 1a).

To examine whether chromosome deletion could be achieved directly by CRISPR/Cas9 editing in established mouse embryonic stem (ES) cells, we designed a sgRNA that targeted the locus consisting of more than 50 repeats of an RNA-binding motif gene on the $\mathrm{Y}$ chromosome (Rbmy1a1), which are clustered in the short arm [10]. Alternatively, we targeted the spermiogenesis-specific transcript on Y 2 (Ssty2) [11] that contains repeated gene sequences scattered in the long arm (Fig. 1a). One day after transfecting mouse ES cells of XY genotype with plasmids expressing Cas9, Y chromosome-targeting sgRNAs, and mCherry, we sorted mCherry-positive ES cells by FACS and cultured them on feeder cells (Fig. 1b). To detect whether the Y chromosome was eliminated, we performed DNA-FISH (fluorescence in situ hybridization) analysis 3 days later using a whole-chromosome probe for the $\mathrm{Y}$ chromosome and near-centromere probe XqA7.3 for the X chromosome (see "Methods") on the transfected cells. We found that about 30 and $50 \%$ of ES cells targeted for Rbmy1a1 and Ssty2, respectively, had no Y chromosome signals, indicating $\mathrm{Y}$ chromosome elimination in some culture cells (Fig. 1c, d). This efficiency of chromosome deletion was much higher than that achieved via spontaneous chromosome loss or Cre-mediated chromosome deletion in previous studies $\left(<10^{-4}\right)[2,3]$. To further confirm Y chromosome elimination, single clones derived from transfected cells were randomly picked, expanded, and genotyped. We found the absence of the Y-specific gene Sry (on the short arm of the Y chromosome) in 4/18 (22\%) clones with Ssty2 targeting and 10/52 (19\%) clones with Rbmy1a1 targeting (Fig. 1e, f). Karyotyping of Srynegative ES cells showed 39 instead of 40 chromosomes (Fig. 1g; Additional file 1: Table S3), and DNA FISH and whole genome sequencing (WGS) further confirmed the complete deletion of the Y chromosome (Fig. 1c, h, i).

To test whether the Y chromosome could be eliminated in vivo by CRISPR-Cas9 editing, we delivered the sgRNA-Ssty2-EGFP construct targeting the Y chromosome to E14.5 mouse brain via in utero electroporation (Additional file 1: Figure S1a). Two days after electroporation, we sorted EGFP-positive cells in the male brain by FACS and performed DNA-FISH (Additional file 1: Figure S1b). We found that about $40 \%$ of EGFP-positive cells showed no Y chromosome signal (Additional file 1: Figure S1c-e). By contrast, only $1 \%$ of wild-type (WT) cells and $8 \%$ of EGFP-negative cells in the brain contained no Y chromosome signal (Additional file 1: Figure S1c-e). These results indicate that the $\mathrm{Y}$ chromosome could be efficiently eliminated in vivo.

Together, these results indicate that the $\mathrm{Y}$ chromosome could be selectively eliminated in vitro and in vivo by CRISPR/Cas9-mediated multiple cuts at chromosomespecific repeated sequences.

\section{Generation of a mouse model with Turner syndrome by $Y$ chromosome elimination}

Next we examined whether this method could be applied to generate animal models for aneuploidy, such as Turner syndrome [1]. We first injected Cas9 mRNA and two specific sgRNAs that targeted the Rmbyla1, Ssty1, or Ssty2 locus into individual mouse zygotes, and injected zygotes were then cultured to the blastocyst stage (Fig. 2a, b). Gene-edited embryos showed normal development compared to untreated embryos (without injection of Cas9 mixture) or embryos treated with two sgRNAs targeting only a single-copy gene $(K d m 5 d)$ on the $\mathrm{Y}$ chromosome, with a similar blastocyst rate (Fig. 2c). To detect whether the $\mathrm{Y}$ chromosome was indeed eliminated, we performed DNA-FISH analysis on injected 


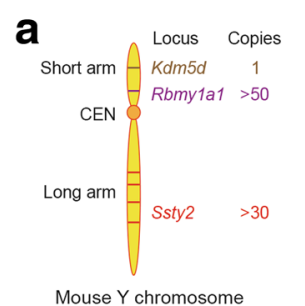

Mouse $Y$ chromosome
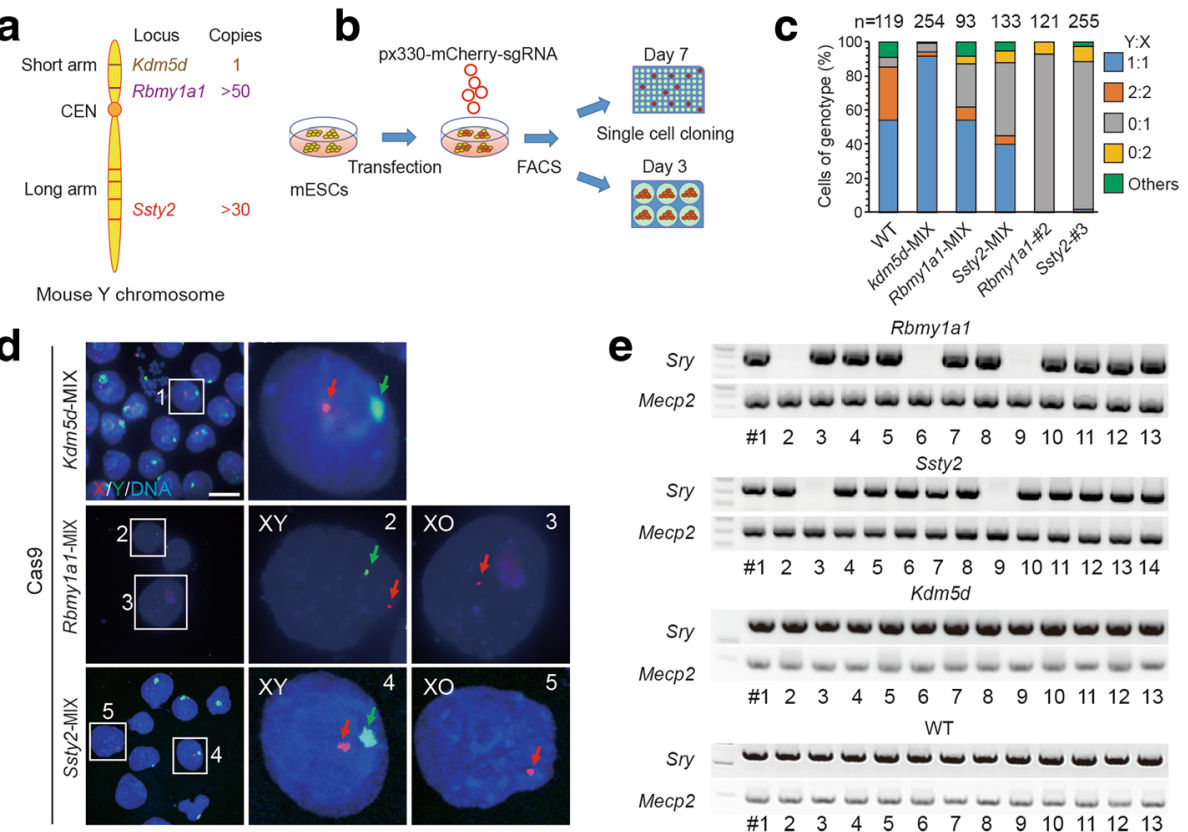

e sry

Mecp2
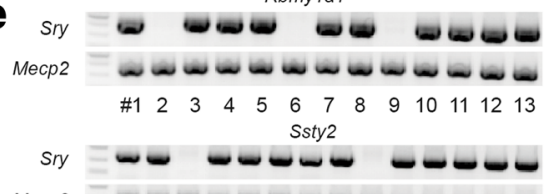

$\begin{array}{lllllllllllll}\# 1 & 2 & 3 & 4 & 5 & 6 & 7 & 8 & 9 & 10 & 11 & 12 & 13\end{array}$ Ssty2

Mecp2

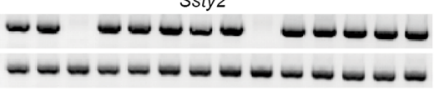

$\begin{array}{lllllllllllll}\# 1 & 2 & 3 & 4 & 5 & 6 & 7 & 8 & 9 & 10 & 11 & 12 & 1314\end{array}$ Kdm5d

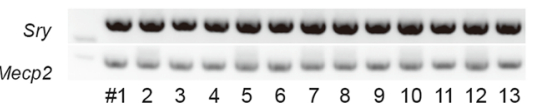
WT

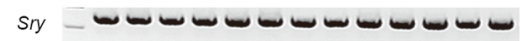

Mecp2

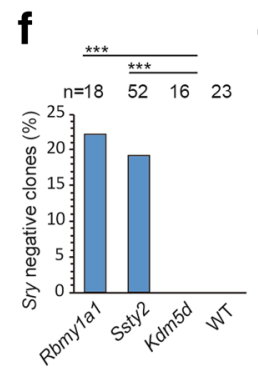

g

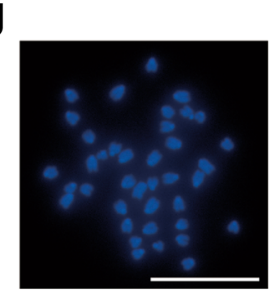

$\mathbf{i}$ बิ
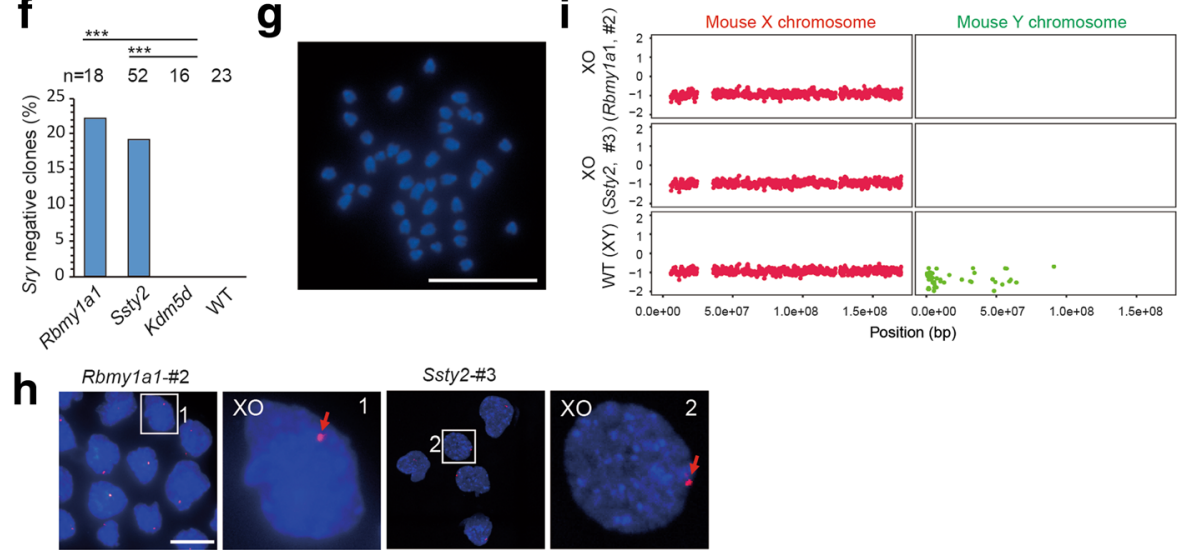

Ssty2-\#3
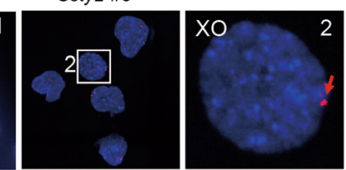

Fig. 1 CRISPR/Cas9-mediated Y chromosome elimination in vitro. a Targeted gene loci in the Y chromosome: Rbmy $1 a 1$, clustered in the short arm; Ssty2,scattered in the long arm; Kdm5d, control gene. b Experimental design. Mouse embryonic stem cells ( $m E S C s$ ) of XY genotype were transfected with plasmids expressing Cas9, Y chromosome-targeting sgRNAs, and mCherry. One day later, mCherry-positive mESCs were sorted by FACS and cultured in six wells for DNA-FISH analysis or 96 wells for single-cell cloning and genotyping. c Stacked bar graphs showing results of DNA-FISH analysis on the gene-edited mESCs. Percentages of cells (including dividing cell, Y:X=2:2 or 0:2) exhibiting different genotype ratios. WT are wild-type, untransfected cells; $n$ is the sample size of counted cells. $\mathbf{d}$ Representative DNA-FISH analysis of mixed ESCs targeted at Rbmy 1a1, Ssty2, or Kdm5d. Green, FITC-labeled whole-chromosome probe for Y chromosome; red, Texas red-labeled X chromosome probe for XqA7.3; blue, Hoechst 33342-labeled DNA. Green arrows indicate Y; red arrows indicate X. Numbered squares indicate single cells shown at a higher resolution in the right panels. Bar, $20 \mu \mathrm{m}$. e Genotyping analysis of ESC clones from Rbmy $1 a 1$ and Ssty2 targeting. Controls: Kdm5d targetted and untransfected (WT) cells. Sry and Mecp2 are located on Y and X, respectively. $\mathbf{f}$ Efficiency of Y chromosome elimination by Rbmy $1 a 1$ and Ssty2 targeting. The experimental groups (Rbmyla1 and Ssty2) showed more Sry-negative clones than the control group (Kdm5d and WT; ***P<0.001, ${ }^{*} P<0.01,{ }^{*} P<0.05$, Chi-square test). g Karyotyping of Sry-negative ESCs showed 39 instead of 40 chromosomes. Bar, $20 \mu \mathrm{m}$. h Representative DNA-FISH analysis of single ESC clones targeted at either Rbmy1a1 or Ssty2. Bar, 20 mm. i Whole genome sequencing showing Y chromosome elimination of XO ESCs. The XO ESCs (Rbmy1a1, \#2; Ssty2, \#3) showed one copy of X with Y absent. WT, untreated mouse. Vertical axis, copy number; horizontal axis, chromosome number

embryos at the 4- to 16-cell stage. We focused only on male embryos, which were determined by the presence of only a single fluorescent dot for the $\mathrm{X}$ chromosome in each blastomere. A green fluorescent signal for the $\mathrm{Y}$ chromosome probe was absent in some blastomeres of injected male embryos, suggesting the Y chromosome had been eliminated (Fig. 2d). The efficiency of $\mathrm{Y}$ chromosome elimination varied from 40 to $90 \%$ among 
the blastomeres of male embryos from three sets of experiments targeted at three different targeted gene loci (Fig. 2e). Based on the extent of Y chromosome deletion in all blastomeres, the injected male embryos could be classified into three phenotypes: $\mathrm{XY}$ (no $\mathrm{Y}$ deletion), pure $\mathrm{XO}$ ( $\mathrm{Y}$ deletion in all blastomeres), and $\mathrm{XY} / \mathrm{XO}(\mathrm{Y}$ deletion in some blastomeres) (Fig. 2f). These results indicate that complete or mosaic $\mathrm{Y}$ chromosome elimination in mouse embryos could be achieved by this method.

In parallel to the above studies, we transferred the injected zygotes into recipient female mice and obtained newborn mice at similar birth rates compared to control embryos, indicating no developmental defect was induced by the gene editing (Fig. 3a). Interestingly, most of the newborn mice were female, as judged by the presence of female genitals and nipples, with the percentage of females ranging from $79-90 \%$ in experiments targeting three different gene loci (Fig. 3b). As a control, mice generated by Cas 9 and sgRNA targeting $K d m 5 d$ or dCas9 (nuclease-dead Cas9) and sgRNA targeting Ssty2 exhibited normal sex ratios (Fig. 3b). To test whether some of these female mice with Rbmy1a1, Ssty1, or Ssty 2 targeting were derived from male embryos that were transformed into females via Y chromosome elimination, we performed karyotyping of tail tissues or bone marrow of all female mice generated by gene editing and found that $26-60 \%$ of these mice indeed had 39 instead of 40 chromosomes (Fig. 3c, d; Additional file 1: Table S3). DNA-FISH analysis further confirmed that the missing chromosome was indeed the Y chromosome (Fig. 3e). Furthermore, FISH analysis also showed that some mice exhibited XO and XY phenotypes in different cells of the same mouse, indicating mosaicism in Y deletion (Fig. 3e, f). Further confirmation of complete Y chromosome elimination in $\mathrm{XO}$ mice was provided by PCR genotyping,

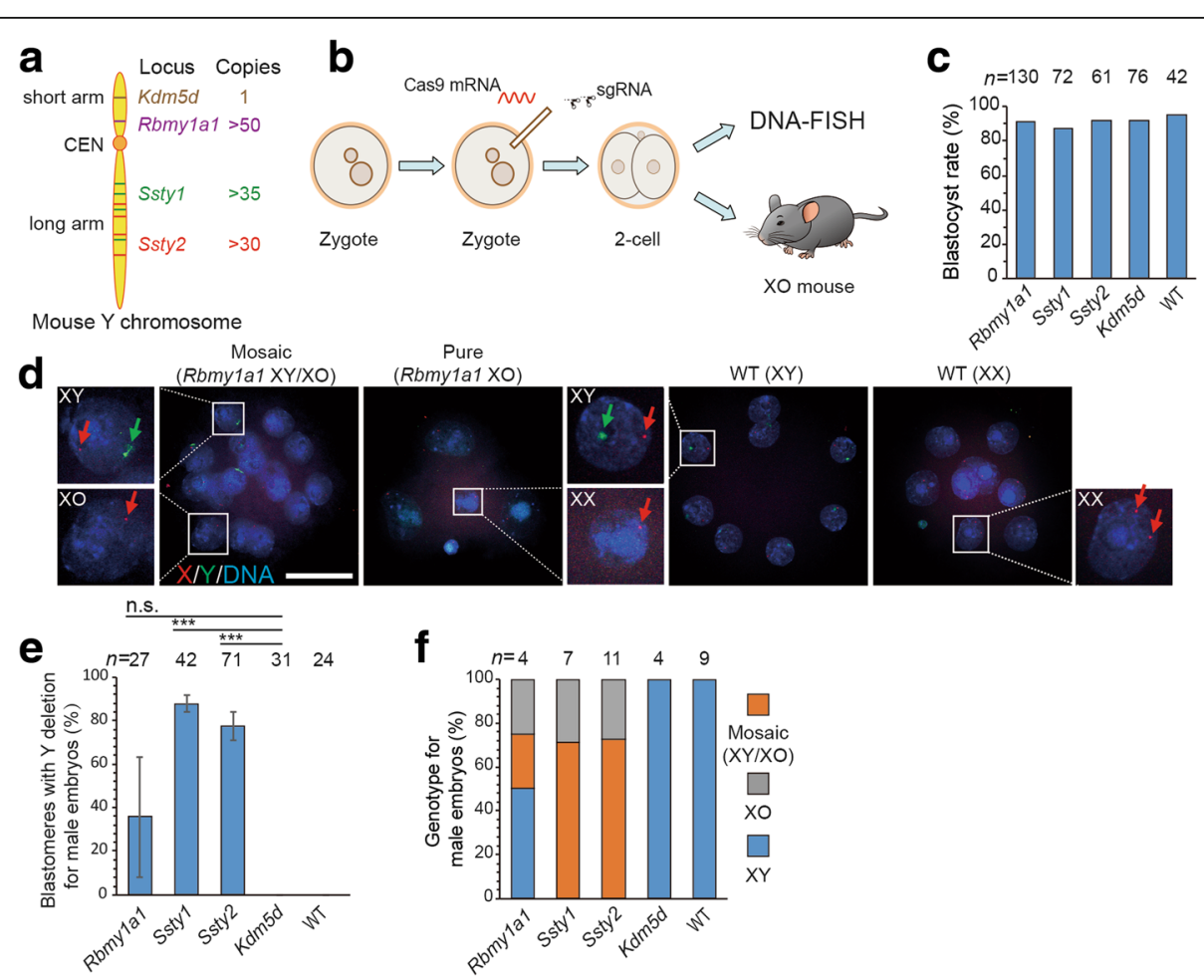

Fig. 2 Elimination of the $Y$ chromosome in zygotes by CRISPR/Cas9-mediated gene editing. a Targeted gene loci in the $Y$ chromosome: Rbmy1a1, clustered in the short arm; Ssty1, Ssty2, scattered in the long arm; Kdm5d, control gene. b Experimental design. Cas9 mRNA and two specific sgRNAs that targeted the Rmby 1a1, Ssty1, Ssty2, or Kdm5d locus were injected into individual mouse zygotes, which were further cultured to 4- to 16-cell embryos for DNA-FISH analysis or transferred into recipients. WT, embryos without injection of Cas9 mixture. c Blastocyst rate of embryos generated by gene editing. The experimental group (Rbmy1a1, Ssty1, Ssty2) showed no difference compared to the control group (Kdm5d or WT); $n$ is the sample size of injected embryos. $\mathbf{d}$ Representative DNA-FISH analysis of 4- to 16-cell embryos after Rbmy 1 a 1 targeting. Green, FITC-labeled whole-chromosome probe for $Y$ chromosome; red, Texas red-labeled X chromosome probe for XqA7.3; blue, Hoechst 33342-labeled DNA. Green arrows, Y; red arrows, X. WT male embryo (XY), one green signal and one red dot; WT female embryo (XX), two red dots, no green signal; pure $X O$ embryo $(X O)$, one red dot, no green signal; mosaic embryo $(X Y / X O)$, co-existing $X O$ and $X Y$ genotype in the blastomeres from the same embryo. Insets: single blastomeres shown at higher resolution. Bar, $50 \mu m$. e, $\mathbf{f}$ Results of DNA-FISH analysis of the ratio of blastomeres with $Y$ deletion $(\mathbf{e})$ and sex chromosomal genotype $(\mathbf{f})$ in each male embryo at 4- to 16-cell stage. Experimental group, Rbmy1a1, Ssty1, Ssty2; control group, Kdm5d and WT (***P<0.001, not significant $>0.05, t$ test). $n$ represents the sample size of blastomeres in e and sample size of embryos in $\mathbf{f}$. Error bars represent SEM 
which showed the absence of ten chromosome-specific genes, located in both the short- and long-arm (Fig. 4a, c). In addition, WGS of XO mice also confirmed complete elimination of the Y chromosome (Fig. 4d). Together, these results showed that the Y chromosome could be efficiently eliminated by CRISPR/Cas9-mediated targeting on clustered repeated gene sequences of Rmbyla1 or scattered repeated gene sequences of Ssty1 or Ssty2.

Compared to the siblings with the $\mathrm{XX}$ karyotype, $\mathrm{XO}$ mice obtained by our gene-editing approach showed normal body weight (Fig. 4e, h; Additional file 1: Table S4), consistent with previous reports [12-14]. However, $\mathrm{XO}$ mice or $\mathrm{XO} / \mathrm{XY}$ mosaic mice from an inbred $\mathrm{C} 57 \mathrm{BL} /$ 6 background showed reproductive defects compared to their wild-type counterparts, including the frequency of pregnancy and parturition (Fig. 4f, g; Additional file 1: Table S4), all of which are found in patients with Turner syndrome $[15,16]$. Interestingly, many patients with aneuploidy diseases (e.g., Turner syndrome) often show mosaicism [17]. Our approach is an efficient way to generate aneuploidy mouse models with mosaicism, which is not found in previous models [18].

The above results on Y chromosome deletion were obtained by using two sgRNAs that target repeat sequences. To generalize this method, we further explored whether the Y chromosome could also be selectively eliminated in zygotes with multiple sgRNAs, each targeting a chromosome-specific single-copy sequence. For this purpose, we designed 14 sgRNAs targeting the short-arm of the Y chromosome (Fig. 5a) and injected Cas9 mRNA together with a cocktail of these 14 sgRNAs into mouse zygotes. We found that $94 \%$ of embryos reached the blastocyst stage and $29 \%$ of embryos yielded live births after they were transferred into pseudopregnant mice, with $73 \%$ (69/94) female (76\% showed XX karyotypes, 17\% showed pure XO karyotypes, and a

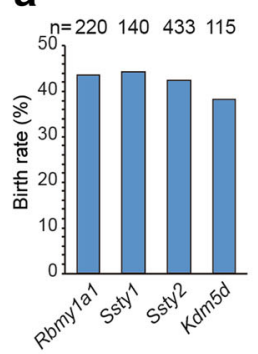

b
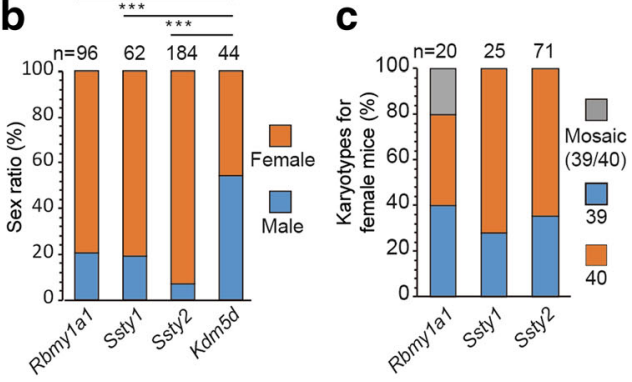

d

$\mathrm{XO}($ Ssty 2 \#5)
11011

$\begin{array}{lllll}1 & 2 & 3 & 4 & 5\end{array}$

11 II 11 t

$\begin{array}{lllll}6 & 7 & 8 & 9 & 10\end{array}$

11 11 II $\mathrm{M}$

$\begin{array}{lllll}11 & 12 & 13 & 14 & 15\end{array}$

11018 ।

$\begin{array}{lllll}16 & 17 & 18 & 19 & \mathrm{X}\end{array}$
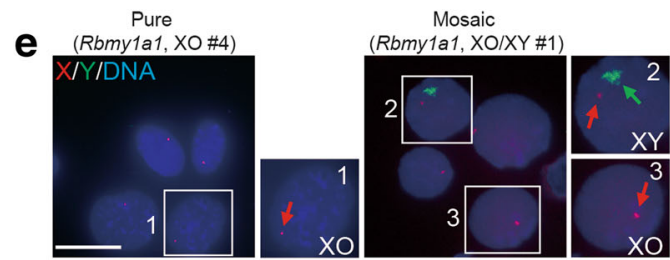

WT $(X Y \# 4)$

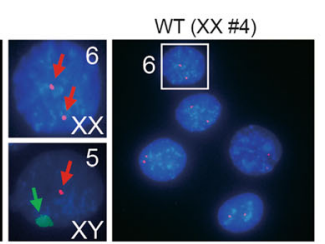

f

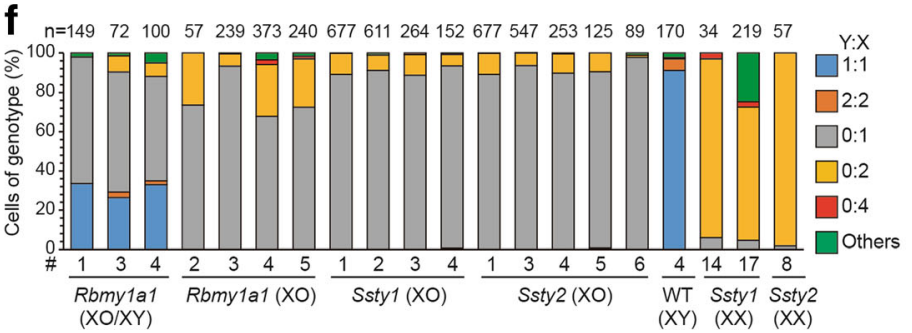

Fig. 3 Generation of mouse model with Turner syndrome by $Y$ chromosome elimination. a Birth rate of gene-edited embryos for $Y$ chromosome elimination. The experimental group (Rbmy1a1, Ssty1, Ssty2) showed no significant difference compared to the control group (Kdm5d). $n$ is the sample size of transferred embryos. b Sex ratio of mice generated by gene editing. The experimental group (Rbmy1a1, Ssty1, Ssty2) showed more mice with female gonads than the control group (Kdm5d or dCas9 and sgRNA targeting Ssty2). $n$ is the sample size of mice generated by gene editing $\left({ }^{* *} P<0.001,{ }^{* *} P<0.01,{ }^{*} P<0.05\right.$, Chi-square test). c Percentage of female mice with different karyotypes. $n$ is the sample size of female mice. $\mathbf{d}$ Representative image of the XO karyotype of a female generated by Ssty2 targeting. e Representative DNA-FISH images of cultured tail fibroblasts derived from female mice with Rbmyla1 targeting, showing XO and XO/XY genotypes. Untreated (WT), male or female mice without gene editing. Green, Y probe; red, X probe for XqC3; blue, DNA. Green arrows, Y; red arrows, X. Numbered squares, single cells shown at a higher resolution in the right panel. Bar, $20 \mu \mathrm{m}$. $\mathbf{f}$ Results of DNA-FISH analysis on the gene-edited female mice. Percentages of cells (including dividing cells, $Y: X=2: 2$ or 0:2) exhibiting different genotype ratios. Data include XO mice (Rbmy1a1, mice 2 to 5; Ssty1, mice 1 to 4; Ssty2, mice 1 to 5; see Additional file 1: Table S3 for corresponding mice) as well as mosaic XO/XY mice (Rbmy1a1, mice 1, 3, and 4). $n$ is the sample size of counted cells 

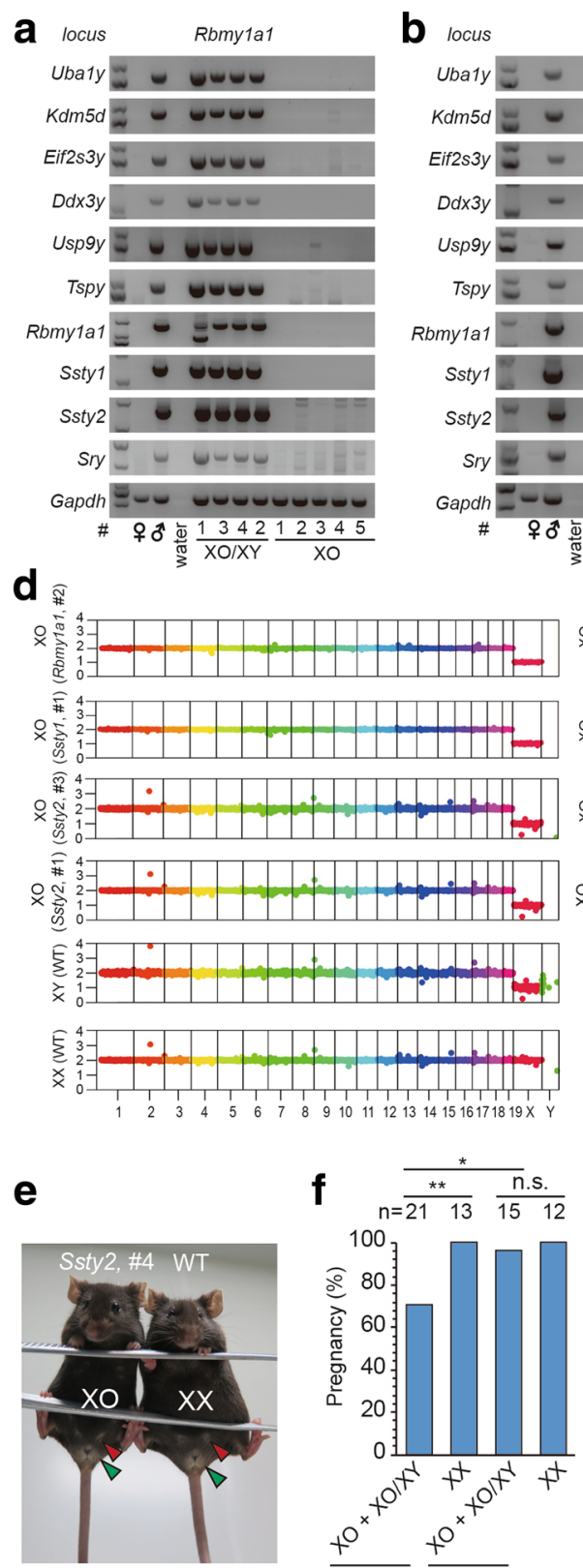

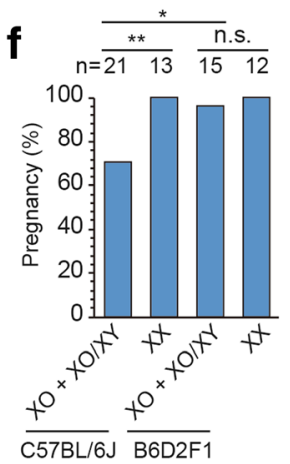

Ssty

(1)

\section{$-$}

\section{$-$}

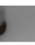

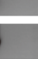
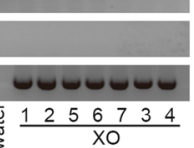

ॠ

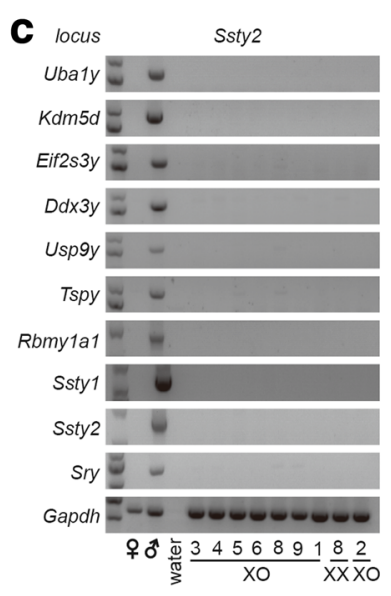

\# Mouse chromosome $X \quad$ Mouse chromosome $Y$

Fig. 4 Identification of a mouse model with Turner syndrome generated by $Y$ chromosome elimination. a-c Rbmy1al (a), Ssty1 (b), Ssty2 (c) targeted mice used for genotyping (listed in Additional file 1: Table S3). The XO pure mice showed no Y chromosome-specific genes, suggesting complete elimination of the Y chromosome. Y chromosome short arm, Ubaly, Kdm5d, Eifs23y, Dxd3y, Usp9y, Tspy, Sry, Rbmy1a1; Y chromosome long arm, Ssty1, Ssty2. Gapdh, control gene in autosome. d WGS showed $Y$ chromosome elimination of XO mice. Histograms of $X$ and $Y$ chromosome are shown in the right panel at a higher resolution. The XO mice (Rbmy1a1, \#2 and Ssty1, \#1) showed one copy of the $X$ chromosome with the Y chromosome absent. Vertical axis, copy number; horizontal axis, chromosome number. e Twelve-week old XO mouse from Ssty 2 XO \#4 showing normal female genitals (green arrowheads) and nipples (red arrowheads). WT, female mice without gene editing. $\mathbf{f}, \mathbf{g}$ Fertility of mice with $Y$ chromosome deletion. The gene-edited $X O$ female mice (number indicated above) were paired with wild-type male mice for over 3 months. The frequency of pregnancy $(\mathbf{f})$ and litter size $(\mathbf{g})$ was determined and compared with those of $X X$ siblings $\left({ }^{* *} P<0.01\right.$, ${ }^{*} P<0.05$, not significant (n.s.), $\mathrm{P}>0.05$, Chi-square test). $\mathbf{h}$ The weight of XO mice (Romy1a1, Ssty1, Ssty2) and the siblings of XX mice. The mice were measured about once a week from 1 week to 9 weeks. Means \pm SEM

7\% showed XY/XO karyotypes) (Fig. 5b-e; Additional file 1: Table S3), as confirmed by genotyping and DNAFISH (Fig. 5f-h; Additional file 1: Table S3). Thus, the Y chromosome could also be selectively eliminated in zygotes by CRISPR/Cas9-mediated multiple-sgRNA targeting at chromosome-specific single-copy sequence sites. 

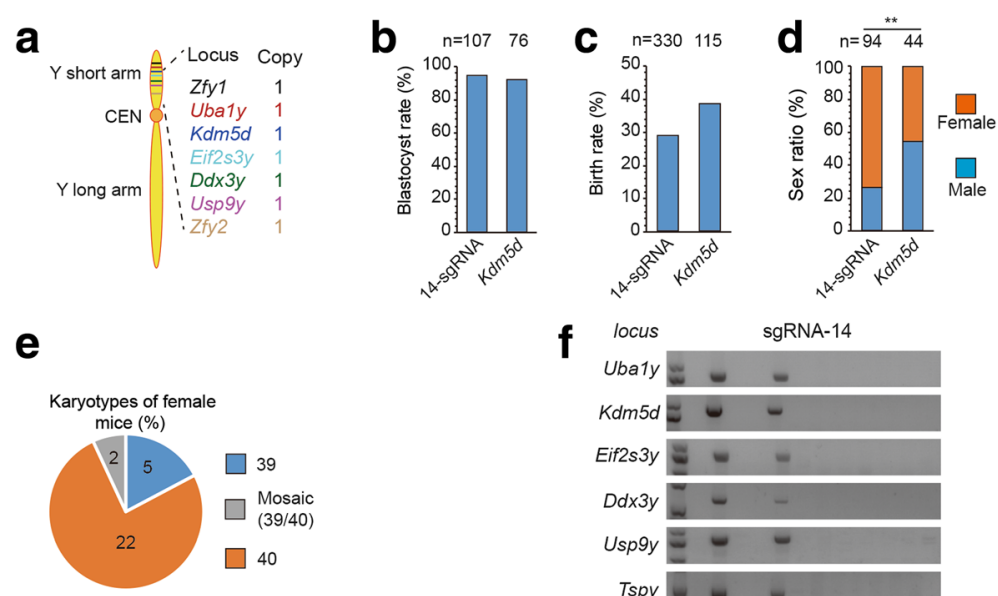

f
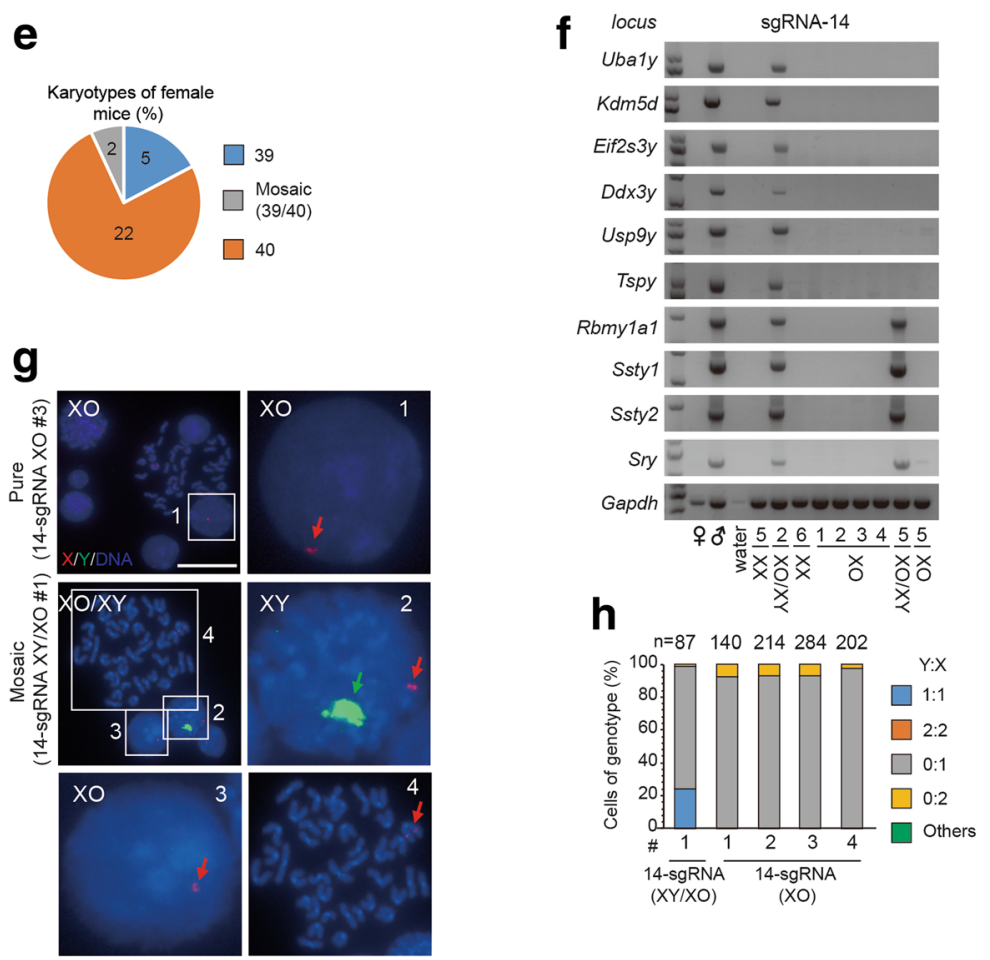

Fig. 5 Elimination of mouse $Y$ chromosome in zygotes with an sgRNA cocktail. a Schema of targeted gene loci in $Y$ chromosome. 7 one-copy genes, each targeted with two sgRNAs, are in the short arm. b, c The blastocyst (b) and birth rate (c) of embryos with 14-sgRNA cocktail for $Y$ chromosome elimination. Control: Kdm5d. ' $n$ ': sample size. $\mathbf{d}$ Sex ratio of the mice generated by gene editing. Experimental group (14-sgRNA cocktail) showed more female mice than control group ( $K d m 5 d)$. ( ${ }^{* *} P<0.001$, ${ }^{* *} P<0.01,{ }^{*} P<0.05$, Chi-square test). e Karyological characteristics of the female mice. $\mathbf{f}$ Genotyping analysis of the female mice obtained by 14-sgRNA targeting. The mice used for genotyping were listed in Additional file 1: Table S3. The mosaic mouse of 14-sgRNA \#2 showed the integrity for the remaining Y chromosome. In contrast, the mosaic mouse of 14-sgRNA \#5 showed the large deletion of short arm in the remaining Y chromosome. The XO pure mice showed no Y chromosome-specific genes, suggesting complete elimination of whole $Y$ chromosome. $\mathbf{g}$ Representative DNA-FISH analysis of the female from 14-sgRNA cocktail targeting. The pure $X O$ mouse of 14-sgRNA-XO \#3 showed the absence of $Y$ chromosome. The mosaic mouse of 14-sgRNA-XY/XO \#1 showed co-existing XO and XY genotype in the cells from the same mouse. Green: a whole $Y$ chromosome probe; red: $X$; blue: DNA. Arrows: $Y$ or $X$ chromosome. Numbered squares: single bone marrow cells shown at a higher resolution on the right or down panel. Bar, $20 \mu \mathrm{m}$. $\mathbf{h}$ Stacked bar graphs of data from DNA-FISH analysis of gene-edited female mice. Percentages of cells (including dividing cells, $Y: X=2: 2$ or $0: 2$ ) exhibiting different ratio of genotype. Experimental group: 14-sgRNA-XO \#1 to \#3; 14-sgRNA-XY/XO \#1, \#2. Control male mice: (WT-XY \#4); control female mice: (Ssty1-XX \#14, \#17, Ssty2-XX \#8). 'n': sample size of counted cells

This approach offers a potential way to use chromosomespecific single nucleotide polymorphisms for chromosome removal without affecting homologous chromosome [19].

\section{Generation of mouse model with Turner Syndrome by $X$ chromosome elimination}

Next, we examined whether the same CRISPR/Cas9-mediated genome editing could be used to eliminate the $\mathrm{X}$ chromosome. We injected Cas9 mRNA together with a single sgRNA (X-A to X-E) or triple sgRNAs (X-A + B +
$\mathrm{C}$ and $\mathrm{X}-\mathrm{C}+\mathrm{D}+\mathrm{E})$ that targeted at $\mathrm{X}$ chromosomespecific repeated sequences in non-coding DNA sequences (Fig. 6a). We found that triple sgRNAs editing led to serious embryonic lethality, possibly due to large fragment deletion of $\mathrm{X}$ chromosomes, whereas single sgRNA targeting yielded some embryos that reached the blastocyst stage (Fig. 6b). We then transferred 2-cell embryos edited with the single sgRNA (X-B, X-C) to recipient female mice and found that these edited embryos yielded birth rates lower than control embryos, using 


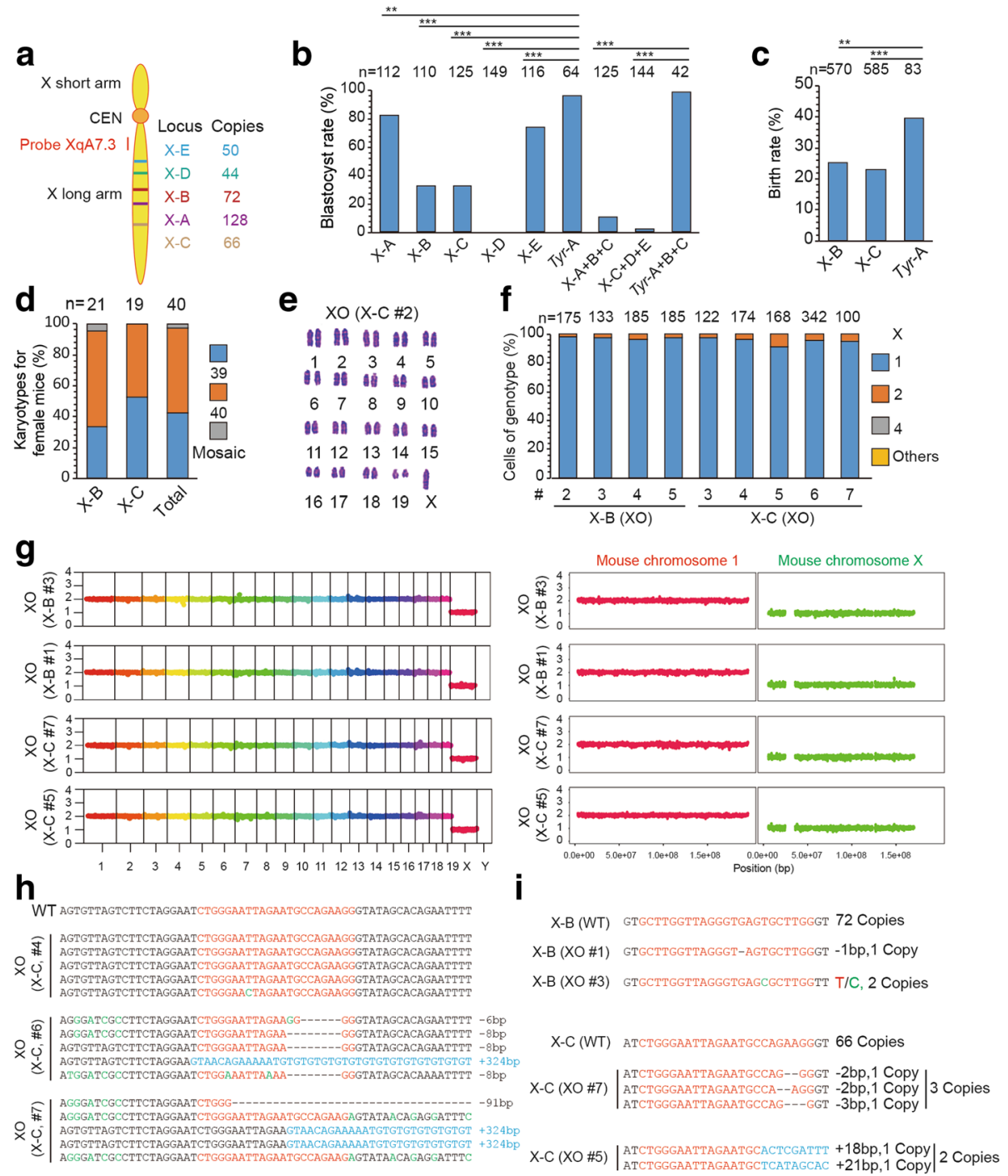

Fig. 6 Generation of mouse model with Turner syndrome by X chromosome elimination. a Targeted loci in the X chromosome. Five sgRNA target sequences (from $X-A$ to $X-E$ ) are $X$ chromosome-specific repeated sequences in non-coding regions. Texas red-labeled probe XqA7.3 is near the centromere. $\mathbf{b}$ Blastocyst rate of embryos generated by gene editing for $X$ chromosome elimination. Embryos edited using triple sgRNAs $(X-A+B+C$ or $X-C+D+E)$ showed embryonic lethality, whereas those edited using a single sgRNA $(X-A, X-B, X-C$, or $X-E)$ reached the blastocyst stage, with a lower blastocyst rate than the control group (Tyr-A or Tyr-A $+B+C$ ). Tyr-A or Tyr-A $+B+C$, a single sgRNA or triple sgRNAs targeting the locus of Tyr, a coat color gene with a single copy on the autosome. $n$ is the sample size of cultured embryos ${ }^{* * *} P<0.001,{ }^{* *} P<0.01$, ${ }^{*} P<$ 0.05, Chi-square test). c Birth rate of embryos gene-edited for $X$ chromosome elimination. The experimental group (X-B, $X-C)$ showed a lower birth rate than the control group (Tyr-A). $n$ is the sample size of transferred embryos ( ${ }^{* *} P<0.001,{ }^{* *} P<0.01,{ }^{*} P<0.05$, Chi-square test). d Percentage of female mice $(X-B, X-C)$ with different karyotypes. Percentages of cells (including dividing cells, $X=4$ ) exhibiting different genotype ratios. $n$ is the sample size of female mice. e Representative image for $X O$ karyotype of a female generated by $X$ - $C$ targeting. $\mathbf{f}$ Representative DNA-FISH analysis of the $X O$ mice from $X-B$ and $X-C$ targeting. $\mathbf{g}$ WGS showing $X$ chromosome elimination of $X O$ mice. The histograms of chromosome 1 and $X$ are shown in the right panel at a higher resolution. The $X O$ mice $(X-B$, mice 1 and $3 ; X-C$, mice 5 and 7$)$ showed one copy of the $X$ chromosome. Vertical axis, copy number; horizontal axis, chromosome number. $\mathbf{h}$ The targeted loci of $X O$ mice $(X-C$, mice 4,6 , and 7$)$ were PCR amplified and pMD-19 T TA cloned for sequencing. Indels occurred in two of three XO mice. i WGS mapping revealed that most copies of clustered repeat sequences by $\mathrm{X}-\mathrm{B}$ or $\mathrm{X}-\mathrm{C}$ targeting were deleted in $\mathrm{XO}$ mice. For $\mathrm{X}-\mathrm{B}$ targeting, 72 copies of target sequences were detected in WT mice. By contrast, only one copy and two copies of target sequence were detected with mutations in X-B XO \#1 and X-B XO \#3, respectively. For X-C targeting, 66 copies of target sequences were detected in WT mice (untreated mice), but only two and three copies of target sequences were detected with mutations in $\mathrm{X}-\mathrm{C} X \mathrm{XO} \# 5$ and $\mathrm{X}-\mathrm{C} X O \mathrm{X}$, respectively

sgRNA that targets at Tyr, a coat color gene with a single copy on autosome (Fig. 6c), suggesting that this gene editing may have induced developmental defects, possibly involving elimination of the single $\mathrm{X}$ chromosome in some male embryos or both $\mathrm{X}$ chromosomes in some female embryos. Gene-edited newborn female 
mice consisted of $42.5 \% \mathrm{XO}$ mice, 55\% XX mice and 2.5\% XO/XX mice (Fig. 6d, e and Additional file 1: Table S3). The absence of $\mathrm{X}$ chromosome in some female mice was confirmed by DNA-FISH and WGS (Fig. 6f, g). As expected, indels or large deletions indeed occurred at targeted non-coding sequences in the remaining $\mathrm{X}$ chromosome in $\mathrm{XO}$ mice (Fig. 6h, i), but these deletions may not induce obvious deleterious effect (Additional file 1: Figure S2a). In principle, these indels and large deletions could be avoided by sgRNAs that target at only one of the two homologus chromosomes, based on single nucleotide polymorphism. Thus, selective X chromosome elimination could also be achieved by CRISPR/ Cas9-mediated gene editing at chromosome-specific repeated sequences, suggesting that this approach could be used for elimination of chromosomes in general.

In addition to establishing a chromosome-deleted mouse model, we have also derived ES cells from blastocysts that were gene-edited by single sgRNA for X chromosome elimination. We obtained 25 out of $52 \mathrm{ES}$ cell lines without a Y chromosome, and examined 10 out of 25 female ES cell lines for further karyotyping. We found that two of them showed a pure XO karyotype, as confirmed by DNA-FISH (Additional file 1: Figure S2b, d). These ES cell lines with distinct karyotypes could be useful for studying the effect of chromosome deletion at the cellular level.

\section{CRISPR/Cas9-mediated gene editing promotes autosome elimination}

We next tested whether an extra chromosome in aneuploid cells could be eliminated by CRISPR/Cas9 editing. We focused on an ES cell line with an extra human chromosome 14 (hChr14), which was established by chromosome transfer (Fig. 7a, b; see "Methods") and known to be stable in cell lines [20]. After FACS analysis, we found that $1.6 \%$ of the mouse ES cells with hChr14 (termed TcH14) exhibited hChr14 loss during every passage (Fig. 7c, d). Using sgRNAs (14-A to 14-F) targeted at repeated sequence sites, we were able to achieve complete elimination of hChr14 in up to $15 \%$ of cells, as indicated by the absence of mCherry expression (Fig. 7c, d). Next, we performed PCR genotyping and DNA-FISH analysis on mCherry-negative clones. We found five out of eight clones from 14-A + F targeting and four out of six clones from 14-F targeting showed complete deletion of hChr14 (Fig. 7e-g). By contrast, clones 14-A + F \#2, \#6, and \#7 and clones 14-F \#3 and \#6 showed the existence of genes in the short arm and hChr14 DNA-FISH probe, indicating incomplete deletion of hChr14 (Fig. 7f, g). Further genotype evidence of the hChr14 deletion was confirmed by WGS, as well as the expression profile of genes unique to hChr14 (Fig. 7h, i). RNA-seq analysis revealed that all hChr14-specific genes showed no expression in clones 14- A + F \#1 and \#8. By contrast, ES cells with hChr14 showed a normal expression profile of genes unique to hChr14 (Fig. 7i). In addition, by injecting these aneuploid cells into oocytes and then injecting Cas9 mRNA and sgRNAs $6 \mathrm{~h}$ later, we found that $13 \%$ of gene-edited blastocysts showed no mCherry signal, indicating complete deletion of hChr14 (Additional file 1: Figure S3a-c). Moreover, we found this method could also be applied to promote human chromosome 7 (hChr7) loss in human cancer cell line HT-29, which contains four hChr7s in most cells (Additional file 1: Figure S4), and extra human chromosome 21 (hChr21) loss in aneuploid mouse ES cell lines (Fig. 8a-e) derived from mice with Down syndrome (DS; Tc1) [21]. Notably, we observed CRISRP/Cas9-mediated multiple DNA cleavages could also produce chromosome rearrangement in cancer cells (Additional file 1: Figure S4e). Finally, we tested whether hChr21 could be selectively deleted via this approach in human iPSCs with trisomy 21 derived from DS patients (ATCC ${ }^{\ominus}$ ACS $-1003^{\mathrm{Tw}}$ ). DS iPS cells were transfected with two sgRNAs (21-A and 21-B, containing 49 and 24 cleavage sites, respectively) targeting hChr21-specific repeated sequence sites (Fig. 8a, b). Transfected cells were sorted and analyzed by DNAFISH with a centromere (CEN) probe for hChr21. We found that $15.0 \%$ of cells showed two hChr21 probe signal dots (Fig. 8f-h). As a control, only $6.9 \%$ of cells transfected with sgRNA containing only one cleavage site on hChr21 showed two hChr21 probe signal dots (Fig. 8f-h).

Taken together, these results indicate that multiple CRISPR/Cas9-induced DNA cleavages could promote targeted autosome loss in aneuploid ES cells, as well as cancer cells.

\section{Off-target effects of CRISPR/Cas9-mediated chromosome elimination}

We next examined whether CRISPR/Cas9-mediated chromosome elimination induces off-target effects in cells and animals. We first analyzed the off-target effects for each sgRNA in seven to nine female mice obtained by $\mathrm{Y}$ chromosome elimination (see Additional file 1: Table S3 for corresponding mice). DNA sequencing of PCR products amplified from these genomic sites showed that mutation rarely occurred at all these loci except Ssty1-A (Additional file 1: Figure S5a). We next analyzed off-target sites with up to five mismatches based on WGS described above, including eight mice with $\mathrm{Y}$ or $\mathrm{X}$ chromosome deletion, and four cell lines with $\mathrm{Y}$ chromosome deletion or hChr14 deletion. Among 2186 to 26,469 potential off-target sites for each sgRNA, we found only two off-target sites in only one XO mouse (Ssty1 \#1) after several filtering steps as described in previous studies, including ENSEMBL repeats and 


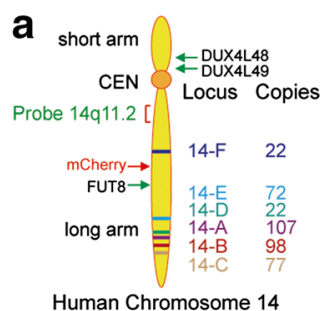

b

Human Chromosome 14

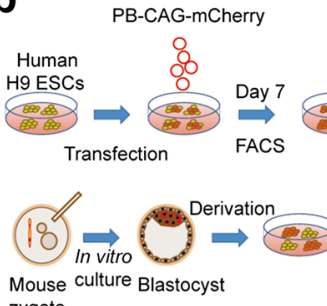
zygote

f
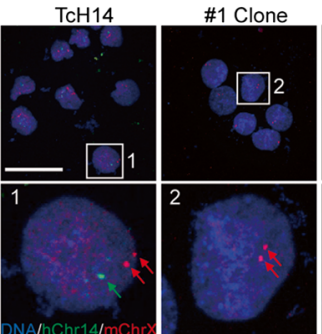

h

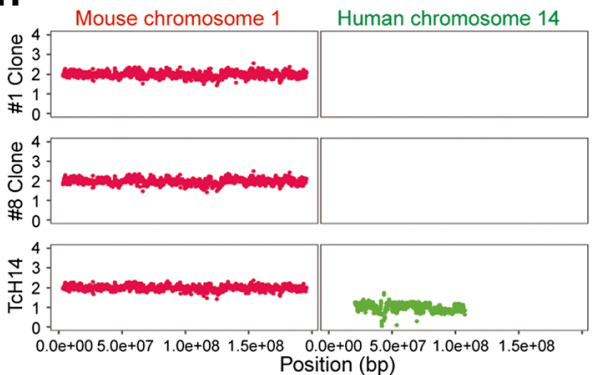

C

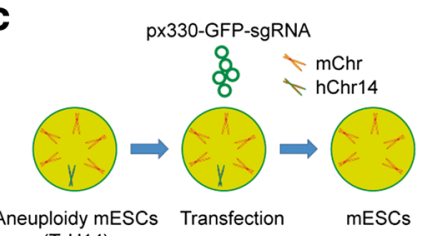
(TcH14)
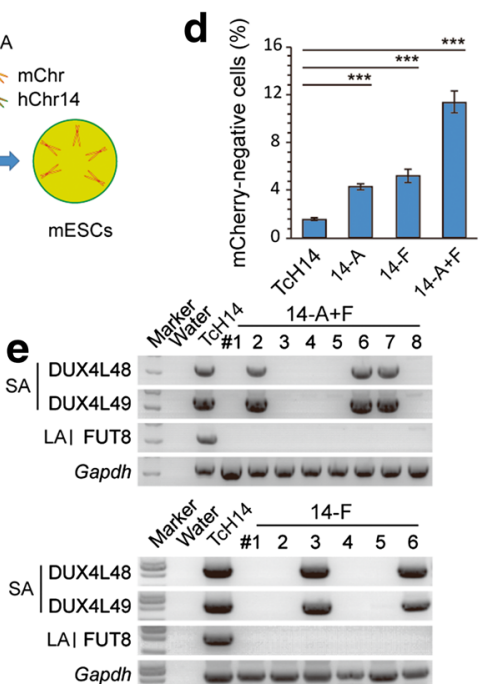

g

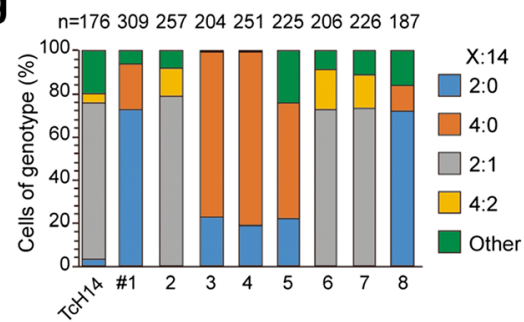

i

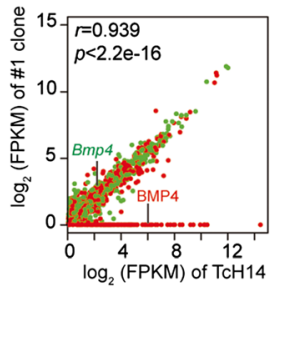

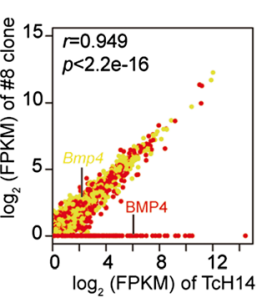

Fig. 7 Elimination of human chromosome 14 in cells by CRISPR/Cas9-mediated gene editing. a Targeted loci in human chromosome 14. Six sgRNA target sequences (from 14-A to 14-F) are specific for human chromosome 14 repeated sequences in the non-coding regions. FITC-labeled probe $14 q 11.2$ is near the centromere. Red arrow, insertion site of PB-CAG-mCherry; green arrows, gene loci for genotyping. $\mathbf{b}$ Establishment of aneuploid mouse ES cells with hChr14 (TcH14). c Experimental design. Aneuploid cells were transfected with plasmids expressing Cas9, chromosome targeting sgRNAs, and mCherry. One day later, GFP-positive ES cells were sorted by FACS and cultured in six wells for DNA-FISH analysis or 96 wells for single cell cloning and genotyping. $\mathbf{d}$ Percentage of mCherry-negative cells after gene editing. TcH14 cells were transfected with px330 plasmid containing different sgRNAs and then sorted by FACS 1 day later. Three days later, transfected TcH14 cells were analyzed by FACS. Data are presented as means $\pm \operatorname{SEM}\left(n=3,{ }^{* *} P<0.001,{ }^{* *} P<0.01,{ }^{*} P<0.05\right.$, Chi-square test). e Genotyping analysis of mCherry-negative cell lines derived from gene editing on TcH14 cells. Note that hChr14 in the cell lines (14-A +F \#2, \#6, \#7; 14-F \#3, \#6) were partial deletions. $\mathbf{f}$ Representative DNA-FISH analysis of clones \#1 and \#8 from 14-A + F targeting. Green, human chromosome 14 probe for 14q11.2; red, X probe for XqA7.3; blue, DNA; green arrow, human chromosome 14; red arrows, mouse X chromosome. Numbered squares, single cells shown at higher resolution in the bottom panels. Bar, $50 \mu \mathrm{m}$. g Results of DNA-FISH analysis on mCherry-negative clones from 14-A + F targeting. Percentages of cells (including dividing cells, X:14=4:2 or 4:0) exhibiting different X:14 ratios. Data include TcH14 mCherry-negative clones (14-A + F\#1 to \#8) as well as control TcH14 mCherry-positive clones. $n$ is the sample size of counted cells. $\mathbf{h}$ The weight of XO mice (X-B, $n=3 ; X-C, n=3)$ and the siblings of XX mice $(n=6)$ was measured about once a week from 1 to 9 weeks. Means \pm SEM. $\mathbf{i}$ RNA-seq analysis of TcH14 cells and cells with chromosome correction. The corrected cell lines (14-A +F \#1 and \#8) showed no gene expression on hChr14 (BMP4 for example) 

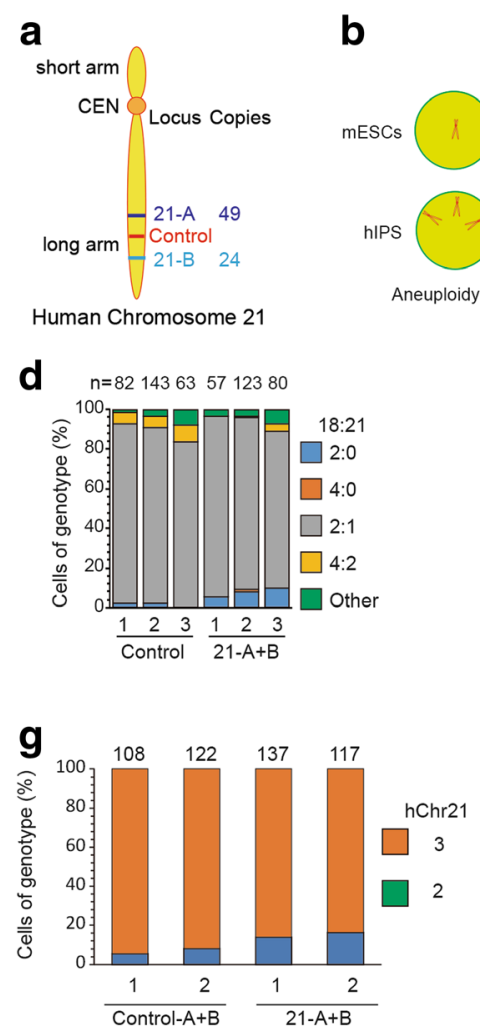
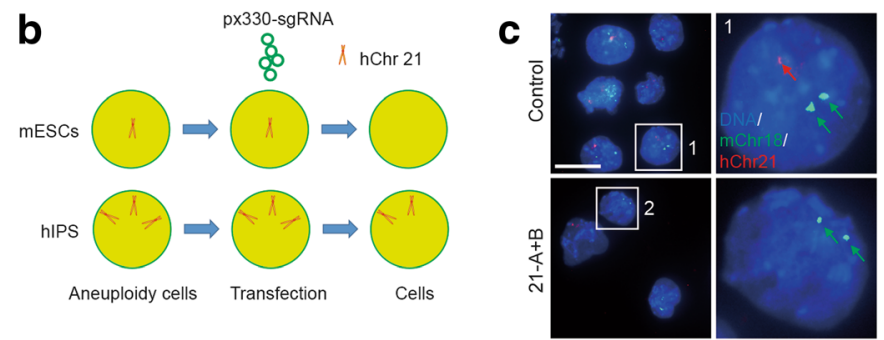

e

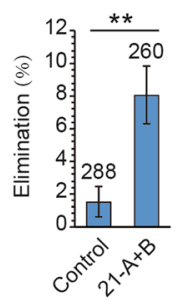

f
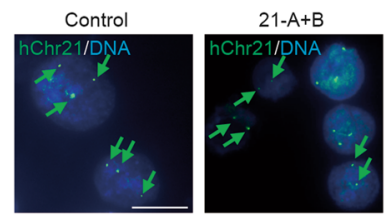

h

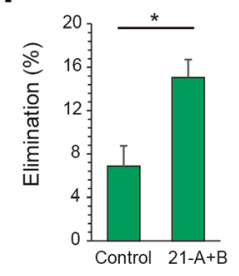

Fig. 8 Elimination of human chromosome 21 in mouse aneuploid cells and DS iPS cells by CRISPR/Cas9-mediated gene editing. a Targeted loci in hChr21. Two sgRNA target sequences (21-A and 21-B) are specific for hChr21 repeated sequences in the non-coding regions. Control sgRNAs (21-NC-A and 21-NC-B) are hChr21-specific sequences with a unique target site. b Experimental design. Aneuploid cells were transfected with plasmids expressing Cas9, chromosome-targeting sgRNAs, and GFP. One day later, GFP-positive ES cells were sorted by FACS and cultured in six wells for DNA-FISH analysis. c Representative DNA-FISH analysis of mixed mouse ES cells targeted at either 21-A + B or control sgRNAs. Red, Texas red-labeled whole-chromosome probe for chromosome 21; green, FITC-labeled mouse chromosome 18qA1 probe; blue, Hoechst 33342-labeled DNA. Green arrows, mChr18; red arrow, hChr21. Numbered squares, single cells shown at higher resolution in the right panels. Bar, $20 \mu \mathrm{m}$. d Results of DNA-FISH analysis on TCH21 cells targeted at either 21-A + B or control sgRNAs (two sgRNAs with one targeting site on hChr21). Percentages of cells (including dividing cells) exhibiting different mChr18: hChr21 ratios. $n$ is the sample size of counted cells. e Efficiency of hChr21 elimination based on DNA-FISH analysis after TcH21 cells targeted at either $21-\mathrm{A}+\mathrm{B}$ or control sgRNAs $\left(n=3,{ }^{*} p<0.01, t\right.$ test). $\mathbf{f}$ Representative DNA-FISH analysis of human iPSCs with trisomy 21 targeted at either 21-A + B or control sgRNAs. Green, FITC-labeled hCHr21 CEN probe; blue, Hoechst 33342-labeled DNA. Arrows, hChr21. Bar, 20 mm. g Results of DNA-FISH analysis on TcH21 cells targeted at either 21-A + B or control sgRNAs (two sgRNAs with one targeting site on hChr21). Percentages of cells (including dividing cells) exhibiting different mChr18: hChr21 ratios. $\boldsymbol{n}$ is the sample size of counted cells. $\mathbf{h}$ Efficiency of $\mathrm{hChr21}$ elimination based on DNA-FISH analysis after DS iPSCs targeted at either 21-A + B or control sgRNAs $\left(n=2,{ }^{* *} P<0.01\right.$, Chi-square test)

microsatellites, variation observed in both mutants and controls (Fig. 9a; Additional file 3: Table S5) [22, 23]. The rest of the mice and cell lines contained no off-target mutations (Fig. 9a; Additional file 3: Table S5). We also examined genomic rearrangements, including deletions, duplications, inversions, and copy number variations, using a strategy described in previous reports [24, 25] and found no rearrangements on the on-target DNA sequence and only two rearrangements in only one $\mathrm{XO}$ mouse (Ssty2 \#1) in the predicted off-targeted sites (Fig. 9b; Additional file 3: Table S5). Furthermore, we examined over 100 metaphase FISH samples among $16 \mathrm{XO}$ mice. We observed that all cells showed XO karyotypes with 39 chromosomes, and none showed fluorescent signals of the $\mathrm{Y}$ chromosome probe, suggesting no obvious ectopic translocation of Y chromosome fragments to other chromosomes (Additional file 3: Figure S6).

Given that the sample sizes of examined cell clones or mice by WGS and FISH were small and the results cannot reveal rare off-target effects in a population of cells or organisms, we employed high-throughput genomewide translocation sequencing (HTGTS) [26] to assess off-target activities of the CRISRP/Cas9 systems. To improve the sensitivity of HTGTS, we introduced magnetic bead-mediated DNA recovery after linear amplification to remove extra biotinylated primers and the final libraries were subjected to Hiseq sequencing (see "Methods" for details). Using CRISRP/Cas9 cutting sites at the 
a

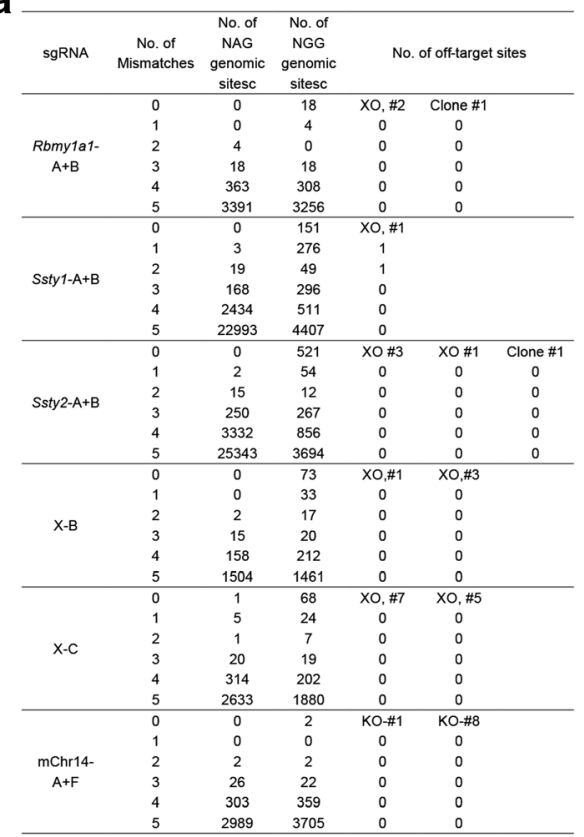

b

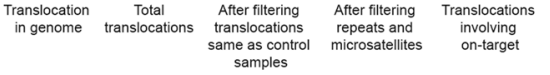

\begin{tabular}{c}
\hline Untreated \\
mouse (XY)
\end{tabular} 696

Untreated

Untreated
mouse $(X X)$$\quad 760$

Untreated
mESC

TcH14 1030

Rbmy1a1 998

$998-337$

Ssty 1
$(\mathrm{XO}, \# 1)$

$(X 0, \#)$
$X-B$
$(X 0 . \# 1)$

$(x-\#)$
$(X 0 . \# 3)$

$x-C$
$(x O, \# 7) \quad 564$
$(x-C)$

$\mathrm{X}-\mathrm{C}$
$(\mathrm{XO}, \# 5) \quad 476$

Ssty2 $\quad 778$

$\begin{array}{ll}\text { (XO \#3) } & \\ \text { Ssty2 } & 768\end{array}$

$(\mathrm{XO \# 1)-768}$

Rbmyla1
(Clone\#1)

Ssty2 518

$\mathrm{TcH} 14$

(\#1) $\quad 438$

$\mathrm{TCH} 14$
$(\#)$

$\begin{array}{llll}996 & & & \\ 54 & & & \\ 30 & & & \\ 98 & 337 & 97 & 0 \\ 406 & 125 & 14 & 0 \\ 400 & 111 & 17 & 0 \\ 62 & 132 & 26 & 0 \\ 564 & 187 & 46 & 0 \\ 46 & 125 & 22 & 0 \\ 778 & 301 & 22 & 0 \\ 768 & 293 & 34 & 2 \\ 452 & 128 & 15 & 0 \\ 18 & 173 & 24 & 0 \\ 438 & 116 & 19 & 0 \\ 560 & 160 & 33 & 0\end{array}$
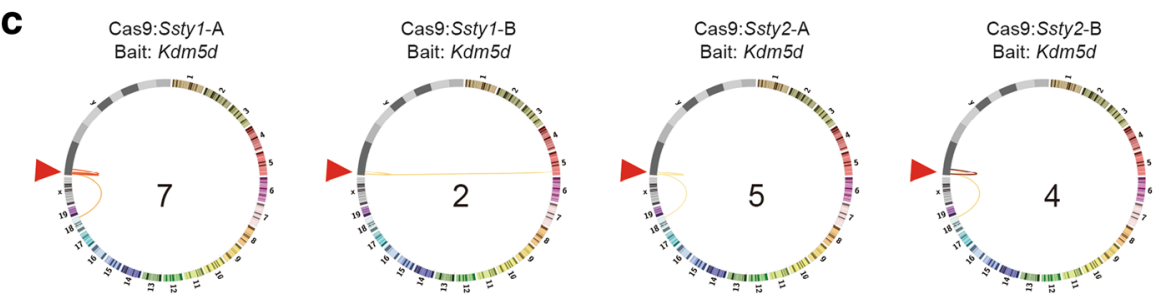

Fig. 9 Off-target analysis in cells and animals with chromosome elimination. a Summary of indels detected by WGS. See Additional file 1: Table S3 for corresponding mice and cell lines. b Summary of genomic rearrangements on the target sequences detected by WGS. c Circos plots showing identified off-target hotspots. Lines link bait site $(K d m 5 d)$ to identified off-target hotspots of indicated CRISPR/Cas9 with a range of color from white to dark red. The higher the frequency, the darker the color. The number of identified off-targets for each CRISPR/Cas9 is shown in the middle of the circus plot. Arrowheads indicate the bait CRISPR/Cas9 Kdm5d site, but note that the bait CRISPR/Cas9 Kdm5d has no detected off-target sites. Three repeats for each treatment (Additional file 1: Table S6 and S7)

$K d m 5 d$ locus as the improved HTGTS (iHTGTS) bait, we barely detected any off-target hotspots for CRISRP/ Cas9 targeting the $K d m 5 d$ site, but did identify several off-target sites for CRISRP/Cas9 targeting the Ssty1 or Ssty2 locus (Fig. 9c; Additional file 1: Tables S6 and S7). The off-target sites located in autosomes were identical to those from the WGS results. Additionally, the majority of the determined off-target hotspots were located in the $\mathrm{Y}$ chromosome, which was invisible to WGS and FISH in Y chromosome-deleted cells. These Y chromosome-containing off-target sites might further promote $\mathrm{Y}$ deletion during chromosome elimination mediated by CRISRP/Cas9, but note that the strongest hotspots harbored only one or two mutation sites in the Cas9-recognition sequences, which should be easily located by bioinformatic prediction. Therefore, strong off-target sites, especially the ones in the same chromosome, should be taken into account during chromosome-elimination using CRISRP/Cas9.
Together, these results indicate that CRISPR/Cas9-mediated chromosome elimination did not induce significant off-target alteration in chromosome-deleted mice and cell lines beyond that expected for CRISPR/Cas9-mediated editing in general $[5,25,27,28]$.

\section{Mechanism of CRISPR/Cas9-mediated targeted chromosome elimination}

Finally, we continued to explore the molecular mechanism underlying CRISPR/Cas9-mediated targeted chromosome elimination. We first checked whether multiple DNA cleavages on the targeted chromosome and cell division are necessary for chromosome elimination. We treated mouse ES cells or embryos with Cas9 and sgRNA targeting $K d m 5 d$ (only one copy on $\mathrm{Y}$ chromosome) or dCas9 and sgRNA targeting Ssty 2 and found no Y chromosome elimination (Fig. 10a, b). To monitor the process of $\mathrm{Y}$ chromosome elimination, we 
stained the injected embryos at different stages from the one- to eight-cell stage. We found no $\mathrm{Y}$ chromosome elimination in one-cell embryos, harvested at $6 \mathrm{~h}$ after sgRNA injection (Fig. 10c). However, Y chromosome elimination was observed at the two-cell stage (65\%) and increased further at the four- to eight-cell stages (85\%) (Fig. 10c). We also tested whether impairing DNA repair by ATM inhibitor KU-55933 could increase the efficiency of chromosome elimination. Mouse ES cells were transfected with Cas9 and sgRNA targeting Ssty 2 and then treated with KU-55933. We found that cells treated with KU-55933 for $48 \mathrm{~h}$ could increase Y chromosome elimination efficiency by 2.65 -fold (Fig. 10d). These results indicate that multiple DNA cleavages on the targeted chromosome, cell division, and DNA repair efficiency are necessary for chromosome elimination.

We next examined whether multiple CRISPR/Cas9mediated cleavages on a targeted chromosome produce micronuclei, resulting from pulverization of chromosomes [29]. After cells were treated with Ssty2 sgRNA, we found that micronuclei-containing Y chromosome was observed around the primary nucleus of cells (Fig. 10e). This chromosome loss may be caused by nuclear exclusion of the targeted chromosome followed by cytoplasmic degradation, a process that requires further study.

\section{Discussion}

A very recent study [30] reported that the Y chromosome could be deleted in ES cells and zygotes by CRISPR/Cas9mediated genome editing. Here we have achieved complete elimination of the Y chromosome by multiple CRISPR/ Cas9-mediated DNA cleavages on the targeted chromosome in ES cells, cells in vivo, and zygotes with high efficiency. Notably, using this approach to eliminate the $\mathrm{X}$ chromosome in mouse embryos with the XX karyotype, one of two homologous $\mathrm{X}$ chromosomes could be efficiently deleted. However, the remaining $\mathrm{X}$ chromosome was also mutated, with indels or fragment deletion in the targeted region. Most chromosome-specific repeated sequences are located in non-coding regions, and thus we could minimize these side effects by targeting the noncoding DNA sequences within small regions $(<2 \mathrm{~kb})$ without obvious biological functions. Alternatively, in principle, these indels and large deletions could be avoided by a

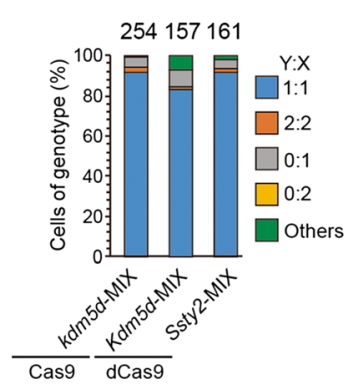

d

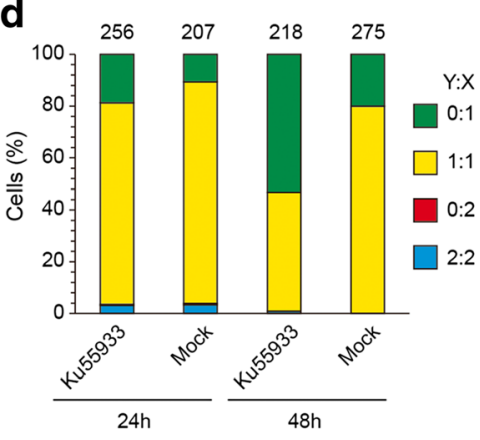

b
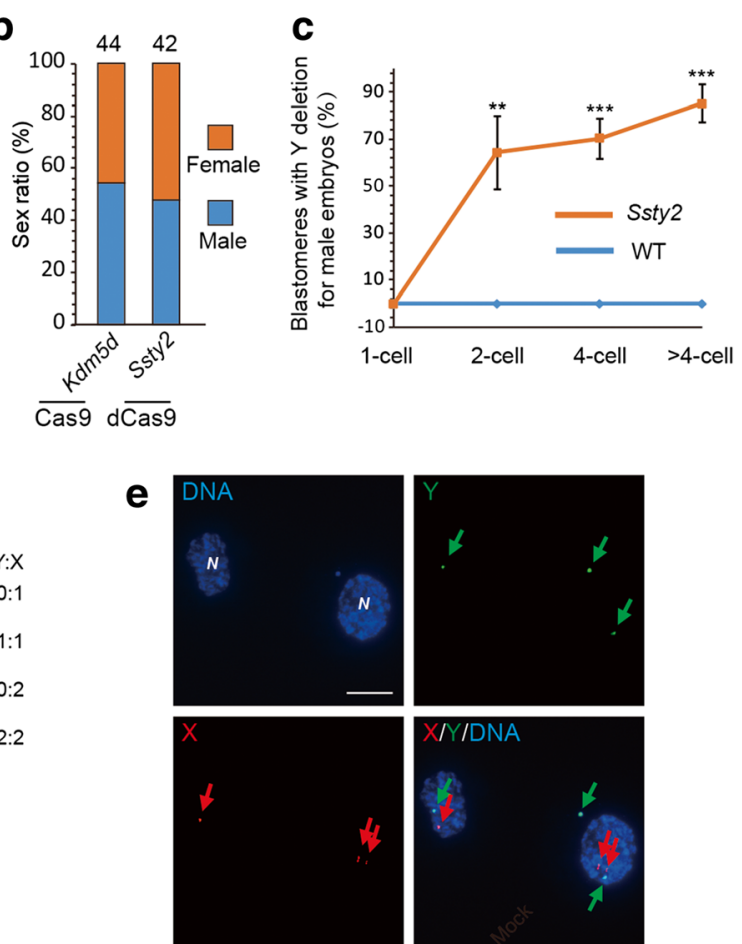

Fig. 10 Mechanism of CRISPR/Cas9-mediated targeted chromosome elimination. a Results of DNA-FISH analysis on the gene-edited ES cells. Percentages of cells (including dividing cell, $Y: X=2: 2$ or 0:2) exhibiting different genotype ratios. $n$ is the sample size of counted cells. $\mathbf{b}$ Sex ratio of mice generated by gene editing. $n$ is the sample size of mice generated by gene editing. c Results of DNA-FISH analysis of the ratio of blastomeres with $Y$ deletion in each male embryo with Ssty2 targeting at different stages. Data are presented as means \pm SEM ( $n=4$ to $12 ;{ }^{*}{ }^{*} P<0.001,{ }^{*} P<0.01,{ }^{*} P<0.05, t$ test). $\mathbf{d}$ Results of DNA-FISH analysis on cells treated with KU-55933 or not. Percentages of cells (including dividing cells, Y:X=2:2 or 0:2) exhibiting different genotype ratios. $n$ is the sample size of counted cells. e Examples of $Y$ chromosome exclusion from nucleus after gene editing. DNA-FISH analysis of mouse ES cells or two-cell embryos with isolated $Y$ chromosome following Ssty2 targeting. Green, Y; red, X; blue, DNA; N, primary nucleus; Arrows: green arrows, Y; red arrows, X. Bar, $20 \mu \mathrm{m}$ 
sgRNAs that target only one of the two homologous chromosomes, based on single nucleotide polymorphism. As shown in Fig. 5, we could delete the Y chromosome with 14 single-target sgRNAs. Nevertheless, reducing the number of sgRNAs and improving the efficiency of chromosome elimination may make this approach more applicable.

We have shown that multiple CRISPR/Cas9-induced DNA cleavages could promote extra hChr14 or hChr21 loss in aneuploid ES cells, as well as hChr7 in human cancer cells. However, we failed to obtain aneuploid mouse ES cells or embryos with autosome deletion (data not shown). We surmise that single-autosome deletion would inhibit cell growth or lead to embryonic lethality. Thus, using aneuploid cells with trisomy autosomes, such as cells from DS patients containing three hChr21s, we detected autosome elimination by CRISPR/Cas9 editing.

Recently, Yang et al. [31] reported that CRISPR/Cas9mediated editing of porcine endogenous retroviruses (PERVs) could remove repetitive sequences (up to 62 copies) but did not delete chromosomes. In comparison with this study, our strategy to eliminate chromosomes is to use sgRNAs targeting repeat sequences in a single chromosome, rather than repeat sequences scattered in many chromosomes. Consistent with this study [31], we observed no obvious off-target mutations or chromosome rearrangements in all examined mouse ES cells and mice with chromosome elimination. Notably, we observed that multiple CRISRP/Cas9-mediated DNA cleavages could produce partial deletion of the targeted chromosome in mice, aneuploid mouse ES cells, and cancer cells, as well as chromosome rearrangement in cancer cells. Furthermore, several off-target sites for CRISRP/Cas9 targeting the Ssty1 or Ssty2 locus were detected by both genomewide off-target assay (iHTGTS) and independent WGS analysis. Therefore, the evaluation of off-target effects by both in silico and in vivo approaches should be taken into account when designing CRISRP/Cas9 systems for chromosome elimination and may be needed before this approach can be used clinically without risk.

Although there are many mouse models for aneuploidy diseases, such as the $\mathrm{XO}$ mouse for Turner syndrome, many features of patients with Turner syndrome could not be well replicated in those mouse models, including the two most common-short stature and premature ovarian failure-which affect over $90 \%$ of recognized patients $[15,18]$. CRISPR/Cas9-mediated targeted chromosome elimination dramatically transforms our ability to generate disease models in diverse organisms, such as in non-human primates. Moreover, this approach would provide a potential therapeutic approach to cure aneuploidy diseases, including DS, Klinefelter syndrome, and XYY syndrome [3, 4, 21, 32-36].

Aneuploidy is a hallmark of cancer [37], and although it can impair cell proliferation and change cell metabolism, it could also promote cell growth under selective pressure, in which context it might contribute to tumorigenesis [38, 39]. Compounds such as AICAR, chloroquine, and 17-AAG, which cause lethality only in aneuploid cells, are in clinical trials of their antitumor activity in multiple myeloma and anaplastic large cell lymphoma [40]. CRISPR/Cas9-mediated targeted chromosome elimination offers a new approach for studying aneuploidy in tumorigenesis and a potential treatment strategy against a broad spectrum of human tumors.

To our knowledge, this is the first report on $\mathrm{X}$ and autosome chromosome elimination via genome editing [41-43]. It paves the way for a potential genetic approach to chromosome therapy in vivo.

\section{Methods}

\section{Production of Cas9 mRNA and sgRNA}

Bicistronic expression vector px330 expressing Cas9, mCherry, and sgRNA was digested with BbsI, and the linearized vector was purified using the Universal DNA Purification Kit (Tiangen). A pair of oligos (Additional file 1: Table S8) for each targeting site was annealed, phosphorylated, and ligated to the linearized vector. The T7 promoter was added to the Cas9 coding region by PCR amplification of px260, using primer Cas9 F and R (Additional file 1: Table S8). The T7-Cas9 PCR product was purified using the Universal DNA Purification Kit (Tiangen) and used as the template for in vitro transcription (IVT) using mMESSAGE mMACHINE T7 ULTRA kit (Life Technologies). The T7 promoter was added to the sgRNA template by PCR amplification of px330, using primers listed in Additional file 1: Table S8. The T7-sgRNA PCR product was purified and used as the template for IVT using a MEGA shortscript T7 kit (Life Technologies). Both the Cas9 mRNA and the sgRNAs were purified using a MEGAclear kit (Life Technologies) and eluted in RNase-free water.

\section{One-cell embryo injection}

All animal procedures were performed under the ethical guidelines of the Institute of Neuroscience, Chinese Academy of Sciences. C57BL/6 J or B6D2F1 female mice and ICR mouse strains were used as embryo donors and foster mothers, respectively. Super-ovulated female C57BL/6 J (4 weeks old) or B6D2F1 mice (7-8 weeks old) were mated to C57BL/6 J or B6D2F1 stud males, and fertilized embryos were collected from the oviduct. Cas9 mRNA $(50 \mathrm{ng} / \mu \mathrm{l})$ and sgRNA $(50 \mathrm{ng} / \mu \mathrm{l}$ for each sgRNA in single to three sgRNA injections, $20 \mathrm{ng} / \mu \mathrm{l}$ for each sgRNA in the 14-sgRNA cocktail injection) were injected into the cytoplasm of fertilized eggs with well-recognized pronuclei in HCZB medium containing $10 \mu \mathrm{g} / \mathrm{ml}$ Cytochalasin $\mathrm{B}(\mathrm{CB})$. The injected zygotes were cultured in $\mathrm{KSOM}$ with amino acids at $37{ }^{\circ} \mathrm{C}$ under $5 \% \mathrm{CO}_{2}$ in air 
until the two-cell stage by day 1 or blastocyst stage by day 3.5. Thereafter, 20 two-cell embryos were transferred into the oviduct of pseudo-pregnant ICR females at $0.5 \mathrm{dpc}$. The blastocysts were used for deriving ES cells.

\section{Karyotype analysis}

Fibroblasts, bone marrow cells, or ES cells were used for karyotyping. Fibroblasts were derived from mouse tails, which were cut into small pieces and cultured for 7 days. Then fibroblasts or mouse ES cells were incubated with $200 \mathrm{ng} / \mathrm{ml}$ demecolcine (Sigma) for $1 \mathrm{~h}$. For bone marrow cells, mice were injected with 15-20 $\mu$ g demecolcine per mouse and bone marrow cells were isolated $4 \mathrm{~h}$ later. The fibroblasts, bone marrow cells, or ES cells were resuspended in $0.075 \mathrm{M} \mathrm{KCl}$ at $37{ }^{\circ} \mathrm{C}$ for $10-30 \mathrm{~min}$, followed by carnoy's fixative ( $25 \%$ acetic acid in methanol) for 30 min cell plating on pre-cleaned slides. For chromosome number counting, the slides were stained with Hoechst 33342. For G banding, the slides were incubated with $0.025 \%$ pepsin and then stained with Giemsa for $15 \mathrm{~min}$. More than ten metaphase spreads were analyzed.

\section{DNA-FISH}

Fibroblasts, mouse ES cells, bone marrow cells, or HT29 cells were harvested, incubated in $0.075 \mathrm{M} \mathrm{KCl}$, and then fixed in 3:1 methanol:glacial acetic acid $(\mathrm{v} / \mathrm{v})$ at $4^{\circ}$ $\mathrm{C}$, and dropped onto microscope slides. For embryos, the zona pellucida was removed with Tyrode's acid and collected onto slides after fixation. The slides were aged at $37^{\circ} \mathrm{C}$ overnight, dehydrated through an ethanol series (70, 90, and $100 \%$ ethanol for 5 min each) at room temperature and air-dried, and then denatured in $70 \%$ formamide/2× SSC at $75{ }^{\circ} \mathrm{C}$ for $5 \mathrm{~min}$ followed by immediate hydration in a $-20{ }^{\circ} \mathrm{C}$ precooled ethanol series (100, 90, and 70\%). The probe (Additional file 4: Table S9, listed below) was denatured in a water bath at $75{ }^{\circ} \mathrm{C}$ for $5 \mathrm{~min}$. The slides were hybridized in a humidified chamber overnight at $37^{\circ} \mathrm{C}$ and rinsed $2 \times 5 \mathrm{~min}$ in $50 \%$ formamide $/ 2 \times$ SSC at $42{ }^{\circ} \mathrm{C}, 2 \times 5$ min in $2 \times \mathrm{SSC}$ at $42^{\circ}$ $\mathrm{C}$ the following day. Finally, the slides were stained with $10 \mu \mathrm{L}$ DAPI-antifade solution and mounted with a coverslip. The samples were captured using an Olympus BX53 fluorescent microscope or Nikon Nie-A1 plus fluorescent microscope. To count probe spots in metaphase spreads, an image of DAPI and a merged image of DAPI and probe signal were analyzed together. For counting probe spots in interphase spreads, spots were counted by two individuals.

\section{Derivation of ES cells}

Morulae or blastocysts were selected to generate ES cell lines. The zona pellucida was removed using acid Tyrode solution. Each embryo was transferred into one well of a 96-well plate seeded with embryonic fibroblast feeders in ES cell medium supplemented with 20\% knockout serum replacement, $1500 \mathrm{U} / \mathrm{ml}$ leukemia inhibitory factor (LIF), $3 \mu \mathrm{M}$ CHIR99021, and $1 \mu \mathrm{M}$ PD0325901. After 4-5 days in culture, the colonies were trypsinized and transferred to a 96-well plate with a feeder layer in the fresh medium. Clonal expansion of the ES cells proceeded from 48-well plates to six-well plates with feeder cells and then to six-well plates for routine culture.

\section{Derivation of aneuploid ES cells}

Mitotic donor cells were obtained after culturing cells with colcemid $(75 \mathrm{ng} / \mathrm{mL}) 10-12 \mathrm{~h}$ at $37^{\circ} \mathrm{C}$. Cells were sedimented at $1000 \mathrm{rpm}$ for $10 \mathrm{~min}$ and resuspended in $10 \mathrm{ml}$ of chromosome isolation GH buffer. Cells were incubated at $37{ }^{\circ} \mathrm{C}$ in a water bath for $10 \mathrm{~min}$ and then on ice for $5 \mathrm{~min}$. We added $100 \mu \mathrm{l}$ of $10 \%$ Triton X-100 to cells (final concentration is $0.1 \%$ ) and incubated them on ice for $5 \mathrm{~min}$. Cells were then lysed by passing three times through a $23 \mathrm{G}$ needle. The homogenate was centrifuged at $1000 \mathrm{rpm}$ for $10 \mathrm{~min}$. Supernatant was collected into a new tube and chromosomes were spun down at $2500 \mathrm{rpm}$ for $20 \mathrm{~min}$. Chromosomes were resuspended in $1 \mathrm{ml}$ of HCZB. Zygotes were obtained from the oviducts of superovulated female mice after mating. The chromosomes were microinjected into denuded zygotes using a piezo-driven micropipette 3-4 $\mu \mathrm{m}$ in diameter. Injected zygotes were cultured in vitro until $3.5 \mathrm{dpc}$ in KSOM (aa). Details of the derivation of mouse ES cells are described in the "Derivation of ES cells" section.

\section{Cell culture and transfection}

$129 / \mathrm{Sv} \times \mathrm{C} 57 \mathrm{BL} / 6 \mathrm{ES}$ cells were cultured on feeder cells using standard ES cell culture conditions. Cells were transfected with px330 expressing Cas9, mCherry, and sgRNA using Lipofectamine 3000 Reagent (Invitrogen) according to the manufacturer's instructions. Forty-eight hours after transfection, mCherry-positive ES cells were sorted into 96 wells using BD FACS AriaII for further culturing. After 7 days of culturing, the colonies were picked up and expanded for further analysis.

For cell treatment with drugs, the ATM inhibitor KU55933 (number S1092, Selleckchem) was used at $20 \mu \mathrm{M}$. Cells were transfected with plasmids (pX330-mCherrySsty2-A and B) and mCherry-positive mouse ES cells were sorted by FACS $12 \mathrm{~h}$ after transfection. DNA-FISH analysis was performed 24 or $48 \mathrm{~h}$ later.

Human iPSCs were purchased from ATCC (ATCC ACS-1003 $3^{\text {tix }}$ ) and cultured on irradiated mouse embryonic fibroblast (iMEFs) feeder layers in serum-free N2B27LCDM medium as described previously [44]. For 
transfection, cells were dissociated using TrypLE, replated in iMEF-coated 12-well plates, and transfected in suspension with gRNAs, Cas9, and EGFP or mCherry plasmid using Lipofectamine 3000. Twenty-four hours after transfection, $\mathrm{EGFP}^{+} / \mathrm{mCherry}^{+}$cells were sorted and used for DNA-FISH analysis at 7 days post-transfection.

\section{WGS and off-target analysis}

We firstly screened the whole mouse (mm10) and human (hg19) genome for chromosome-specific sgRNAs with our in-house script (https://github.com/pingjie/ findChrCrispr). The sgRNAs for each chromosome given by our software are listed in Additional file 1: Tables S2 and S3.

WGS was carried out using Illumina HiSeq X Ten at mean coverages of $20 \times$. Qualified reads were mapped to the mouse reference genome $(\mathrm{mm} 10)$ by speedseq [45] (https:/github.com/hall-lab/speedseq) with default parameters. FreeBayes (v0.9.10) [46] and LUMPY [24] (https://github.com/arq5x/lumpy-sv) were run on the aligned sequence files (BAM files) for short indel detection and structural variation discovery. We firstly filtered germ-line variants which were the same as variants in the "control" (wild-type) samples (untreated mice-XY, untreated mice-XX, untreated mESCs, and TcH14). These results were then filtered to remove variation which overlapped any UCSC repeat regions and microsatellite sequences. The original bam files (pileup) around each candidate variation site were further examined to eliminate those cases where potentially shared variation with "control" samples were not detected by the variant calling software. Next, the raw variant output was manually inspected to remove variants which overlapped with any of the four wild-type samples. For the short indel variations, homopolymers with unit length greater than $2 \mathrm{bp}$ were also removed [22]. Variations after each filtering step are listed in Additional file 1: Table S5.

To search for rearrangements involving on-target DNA sequences that might have integrated elsewhere in the genome, we detected whole genome translocation cases $[24,25]$ in each sample. After filtering translocations in both mutant and wild-type samples that overlapped with the UCSC repeat regions and microsatellites, the translocations involving the on-target sequences were not observed in any of our mutant samples.

Potential off-target sites of sgRNAs were predicted as previously reported [47] (http://www.rgenome.net/casoffinder/). We extracted all the off-target sites with no more than five mismatches for each sgRNA. We searched the short indel variations within the 23-bp predicted off-target sites, and structural variations within 250 bp up- or downstream of the potential off-target sites (Additional file 3: Table S5).

\section{iHTGTS}

Mouse ES cells were transfected with plasmids carrying indicated CRISPR/Cas9 and were harvested $48 \mathrm{~h}$ later and then digested with Protease $\mathrm{K}$ to extract for genomic DNA. The iHTGTS libraries were prepared following the protocol described previously with minor modifications [26]. Briefly, linear amplification was performed with $20 \mathrm{nM}$ biotinylated primer (biotinCCCATTTGCTATTGTTGACAGGAAACCCACTGCC, by Sangon, Shanghai) for 80 cycles; extra primers were removed by $1.2 \times$ AxyPrep Mag PCR Clean-Up beads (Axygen, US). Locus-specific primer CTTTGGAGTG AATGTCTGCTCC was used for nested PCR. KpnI was used to block germline fragments, and 1.0× AxyPrep Mag PCR Clean-Up beads (Axygen, US) were used to recover the DNA after enzyme blocking. All the iHTGTS libraries were sequenced by Hiseq. Bioinformatic analysis for off-targets followed the protocol described previously [48].

\section{Genotyping}

Genotyping of the mice was performed by PCR using DNA extracted from their tails. Single ES cell clones were genotyped by nested PCR. The single clone was dissolved in DNA lysis buffer $(4 \mu \mathrm{g} / \mu \mathrm{l}$ proteinase $\mathrm{K}$, $0.1 \%$ Triton X 100, and $0.1 \%$ Tween 20 in nuclease-free water). The samples were digested at $55^{\circ} \mathrm{C}$ for $30 \mathrm{~min}$ and then the proteinase $\mathrm{K}$ was inactivated at $95{ }^{\circ} \mathrm{C}$ for $5 \mathrm{~min}$. PCR was performed using specific primers (Additional file 1: Table S8) under the following conditions: $95{ }^{\circ} \mathrm{C}$ for 5 min followed by 35 cycles of PCR $\left(95{ }^{\circ} \mathrm{C}\right.$ for $30 \mathrm{~s}, 58^{\circ} \mathrm{C}$ for $30 \mathrm{~s}$, and $72{ }^{\circ} \mathrm{C}$ for $120 \mathrm{~s}$ ) for mouse. The nested PCR was $95{ }^{\circ} \mathrm{C}$ for $5 \mathrm{~min}$ followed by 25 cycles of PCR $\left(95^{\circ} \mathrm{C}\right.$ for $30 \mathrm{~s}, 62{ }^{\circ} \mathrm{C}$ for $30 \mathrm{~s}$, and $72{ }^{\circ} \mathrm{C}$ for $90 \mathrm{~s}$ ) for mouse ES cells. Secondary PCR was performed using $0.5 \mu \mathrm{g}$ primary PCR product and nested inner primer. PCR was carried out with the same reaction mixture and cycle parameters.

\section{Plasmid DNA delivery into mouse embryos by in utero electroporation}

In utero electroporation (IUE) experiments were performed using ICR mice. IUE was performed as previously described [49]. Briefly, E14.5 pregnant ICR mice were anesthetized with sodium pentobarbitone $(50 \mathrm{mg} / \mathrm{kg})$, and the uterine horns were exposed. Plasmid mixture $(1 \mu \mathrm{L}$; containing the px330-EF1 $\alpha$-EGFP-Ssty2-A sgRNA (1 $\mu \mathrm{g} /$ $\mu \mathrm{l}), \quad \mathrm{px} 330$-EF1 $\alpha$-EGFP-Ssty2-B sgRNA $(1 \mu \mathrm{g} / \mu \mathrm{l}))$ with $0.01 \%$ Fast Green dye (Sigma)) was injected into the embryos' lateral ventricle with a glass micropipette. For electroporation, five pulses with a $50 \mathrm{~ms}$ duration separated by $950 \mathrm{~ms}$ were applied at $32 \mathrm{~V}$ using ECM 830 (BTX). Then the uterine horns were placed back into the abdominal cavity to allow the embryos to continue normal 
development. Forty-eight hours after IUE, the embryos were collected and dissected for FACS.

\section{Conclusion}

In summary, we demonstrated that a single specific chromosome, including a sex chromosome and an autosome, could be selectively eliminated via CRISPRmediated multiple DNA cleavages on the targeted chromosome in culture cells, embryos and in vivo tissues. With the increase of efficiency and specificity, we believe CRISPR/Cas9-Mediated targeted chromosome elimination would be broadly applicable in developing animal models and therapeutic treatments for aneuploidy.

\section{Additional file}

Additional file 1: Supplemental tables and figures. (DOCX $3253 \mathrm{~kb}$ )

Additional file 2: Table S2. Unique and multiple repeated sequences for targeting by a single specific sgRNA. (XLSX 9524 kb)

Additional file 3: Table S5. Summary of genomic variations and filtering steps. (XLSX 19 kb)

Additional file 4: Table S9. Probes used in this work. (DOCX 509 kb)

\section{Acknowledgements}

We thank Mu-ming Poo and Dangsheng Li for comments on the manuscript.

\section{Funding}

This work was supported by National Science and Technology Major Project (2017YFC1001302), CAS Strategic Priority Research Program (XDB02050007, XDA01010409), the MoST863 Program (2015AA020307), NSFC grants (31522037, 31771485), China Youth Thousand Talents Program, Break through project of Chinese Academy of Sciences.

\section{Availability of data and materials}

All of the mapped data are available from the SRA under accession SRP070593.

\section{Authors' contributions \\ E-WZ designed and performed experiments. X-NH and L-YS performed geno- typing, G-band analysis and DNA-FISH. XY and XW derived mouse ES cells and performed ES cell targeting. B-BH, WW, and W-JL performed karyotyping analysis. W-QY transferred embryos. JP and Y.-DS performed data analysis. X- $\mathrm{DH}, \mathrm{WW}, \mathrm{YW}$ and $\mathrm{CT}$ constructed plasmids. JY and JH employed iHTGTS to analyzed the genome-wide off-targets. HY supervised the project and de- signed experiments and HY and M-m. Poo wrote the paper. All authors read and approved the final manuscript.}

\section{Ethics approval and consent to participate}

The use and care of animals complied with the guidelines of the Biomedical Research Ethics Committee at the Shanghai Institutes for Biological Science (CAS).

\section{Competing interests}

The authors declare that they have no competing interests.

\section{Publisher's Note}

Springer Nature remains neutral with regard to jurisdictional claims in published maps and institutional affiliations.

\section{Author details}

Institute of Neuroscience, State Key Laboratory of Neuroscience, Key Laboratory of Primate Neurobiology, CAS Center for Excellence in Brain Science and Intelligence Technology, Shanghai Institutes for Biological Sciences, Chinese Academy of Sciences, Shanghai 200031, China. ${ }^{2}$ State Key Laboratory of Membrane Biology and Minstry of Education Key Laboratory of Cell Proliferation and Differentiation, School of Life Sciences, Peking-Tsinghua Center for Life Sciences, Peking University, Beijing 100871, China. ${ }^{3}$ Key Lab of Computational Biology, CAS-MPG Partner Institute for Computational Biology, Shanghai Institutes for Biological Sciences, Chinese Academy of Sciences, Shanghai 200031, China. ${ }^{4}$ University of Chinese Academy of Sciences, Shanghai 200031, China. ${ }^{5}$ Center for Quantitative Sciences, Vanderbilt University School of Medicine, Nashville, Tennessee 37232, USA. ${ }^{6}$ Shanghai University, Shanghai 200444, China. ${ }^{7}$ College of Animal Science and Technology, Guangxi University, Nanning, Guangxi 530004, China.

Received: 5 July 2017 Accepted: 26 October 2017

Published online: 24 November 2017

\section{References}

1. Driscoll DA, Gross S. Prenatal screening for aneuploidy. N Engl J Med. 2009; 360:2556-62.

2. Matsumura H, Tada M, Otsuji T, Yasuchika K, Nakatsuji N, Surani A, Tada T. Targeted chromosome elimination from ES-somatic hybrid cells. Nat Methods. 2007:4:23-5.

3. Li LB, Chang KH, Wang PR, Hirata RK, Papayannopoulou T, Russell DW. Trisomy correction in Down syndrome induced pluripotent stem cells. Cell Stem Cell. 2012;11:615-9.

4. Jiang J, Jing Y, Cost GJ, Chiang JC, Kolpa HJ, Cotton AM, Carone DM, Carone BR, Shivak DA, Guschin DY, et al. Translating dosage compensation to trisomy 21. Nature. 2013;500:296-300.

5. Wang $H$, Yang $H$, Shivalila CS, Dawlaty MM, Cheng AW, Zhang F, Jaenisch $R$. One-step generation of mice carrying mutations in multiple genes by CRISPR/Cas-mediated genome engineering. Cell. 2013;153:910-8.

6. Yang H, Wang $H$, Shivalila CS, Cheng AW, Shi L, Jaenisch R. One-step generation of mice carrying reporter and conditional alleles by CRISPR/Casmediated genome engineering. Cell. 2013:154:1370-9.

7. Blasco RB, Karaca E, Ambrogio C, Cheong TC, Karayol E, Minero VG, Voena C, Chiarle R. Simple and rapid in vivo generation of chromosomal rearrangements using CRISPR/Cas9 technology. Cell Rep. 2014;9:1219-27.

8. Maddalo D, Manchado E, Concepcion CP, Bonetti C, Vidigal JA, Han YC, Ogrodowski P, Crippa A, Rekhtman N, de Stanchina E, et al. In vivo engineering of oncogenic chromosomal rearrangements with the CRISPR/ Cas9 system. Nature. 2014:516:423-7.

9. Xiao A, Wang Z, Hu Y, Wu Y, Luo Z, Yang Z, Zu Y, Li W, Huang P, Tong X, et al. Chromosomal deletions and inversions mediated by TALENs and CRISPR/Cas in zebrafish. Nucleic Acids Res. 2013;41:e141.

10. Mahadevaiah SK, Odorisio T, Elliott DJ, Rattigan A, Szot M, Laval SH, Washburn LL, McCarrey JR, Cattanach BM, Lovell-Badge R, Burgoyne PS. Mouse homologues of the human AZF candidate gene RBM are expressed in spermatogonia and spermatids, and map to a $Y$ chromosome deletion interval associated with a high incidence of sperm abnormalities. Hum Mol Genet. 1998;7:715-27.

11. Royo H, Polikiewicz G, Mahadevaiah SK, Prosser H, Mitchell M, Bradley A, de Rooij DG, Burgoyne PS, Turner JM. Evidence that meiotic sex chromosome inactivation is essential for male fertility. Curr Biol. 2010;20:2117-23.

12. Burgoyne PS, Evans EP, Holland K. XO monosomy is associated with reduced birthweight and lowered weight gain in the mouse. J Reprod Fertil. 1983:68:381-5.

13. Hunt PA. Survival of $X O$ mouse fetuses: effect of parental origin of the $X$ chromosome or uterine environment? Development. 1991;111:1137-41.

14. Probst FJ, Cooper ML, Cheung SW, Justice MJ. Genotype, phenotype, and karyotype correlation in the XO mouse model of Turner syndrome. J Hered. 2008;99:512-7.

15. Bondy CA. Turner syndrome 2008. Horm Res. 2009;71 Suppl 1:52-6.

16. Donaldson MD, Gault El, Tan KW, Dunger DB. Optimising management in Turner syndrome: from infancy to adult transfer. Arch Dis Child. 2006;91:513-20.

17. Zhong Q, Layman LC. Genetic considerations in the patient with Turner syndrome-45, X with or without mosaicism. Fertil Steril. 2012;98:775-9.

18. Sheppard O, Wiseman FK, Ruparelia A, Tybulewicz VL, Fisher EM. Mouse models of aneuploidy. TheScientificWorldJOURNAL. 2012;2012:214078. 
19. Sachidanandam R, Weissman D, Schmidt SC, Kakol JM, Stein LD, Marth G, Sherry S, Mullikin JC, Mortimore BJ, Willey DL, et al. A map of human genome sequence variation containing 1.42 million single nucleotide polymorphisms. Nature. 2001;409:928-33.

20. Tomizuka K, Shinohara T, Yoshida H, Uejima H, Ohguma A, Tanaka S, Sato K, Oshimura M, Ishida I. Double trans-chromosomic mice: maintenance of two individual human chromosome fragments containing lg heavy and kappa loci and expression of fully human antibodies. Proc Natl Acad Sci U S A. 2000;97:722-7.

21. O'Doherty A, Ruf $\mathrm{S}$, Mulligan C, Hildreth V, Errington ML, Cooke S, Sesay A, Modino S, Vanes L, Hernandez D, et al. An aneuploid mouse strain carrying human chromosome 21 with Down syndrome phenotypes. Science. 2005; 309:2033-7.

22. lyer , Shen B, Zhang W, Hodgkins A, Keane T, Huang X, Skarnes WC. Offtarget mutations are rare in Cas9-modified mice. Nat Methods. 2015;12:479.

23. Yang L, Grishin D, Wang G, Aach J, Zhang CZ, Chari R, Homsy J, Cai X, Zhao $Y$, Fan JB, et al. Targeted and genome-wide sequencing reveal single nucleotide variations impacting specificity of Cas9 in human stem cells. Nat Commun. 2014;5:5507.

24. Layer RM, Chiang C, Quinlan AR, Hall IM. LUMPY: a probabilistic framework for structural variant discovery. Genome Biol. 2014;15:R84

25. Veres A, Gosis BS, Ding Q, Collins R, Ragavendran A, Brand H, Erdin S, Cowan CA, Talkowski ME, Musunuru K. Low incidence of off-target mutations in individual CRISPR-Cas9 and TALEN targeted human stem cell clones detected by whole-genome sequencing. Cell Stem Cell. 2014;15:27-30.

26. Hu J, Meyers RM, Dong J, Panchakshari RA, Alt FW, Frock RL. Detecting DNA double-stranded breaks in mammalian genomes by linear amplificationmediated high-throughput genome-wide translocation sequencing. Nat Protoc. 2016;11:853-71.

27. Hsu PD, Scott DA, Weinstein JA, Ran FA, Konermann S, Agarwala V, Li Y, Fine EJ, Wu X, Shalem O, et al. DNA targeting specificity of RNA-guided Cas9 nucleases. Nat Biotechnol. 2013;31:827-32.

28. Li W, Teng F, Li T, Zhou Q. Simultaneous generation and germline transmission of multiple gene mutations in rat using CRISPR-Cas systems. Nat Biotechnol. 2013;31:684-6.

29. Crasta K, Ganem NJ, Dagher R, Lantermann AB, Ivanova EV, Pan Y, Nezi L, Protopopov A, Chowdhury D, Pellman D. DNA breaks and chromosome pulverization from errors in mitosis. Nature. 2012;482:53-8.

30. Adikusuma F, Williams N, Grutzner F, Hughes J, Thomas P. Targeted deletion of an entire chromosome using CRISPR/Cas9. Mol Ther. 2017;25(8):1736-8.

31. Yang L, Guell M, Niu D, George H, Lesha E, Grishin D, Aach J, Shrock E, Xu W, Poci J, et al. Genome-wide inactivation of porcine endogenous retroviruses (PERVs). Science. 2015;350:1101-4

32. Long C, Amoasii L, Mireault AA, McAnally JR, Li H, Sanchez-Ortiz E, Bhattacharyya S, Shelton JM, Bassel-Duby R, Olson EN. Postnatal genome editing partially restores dystrophin expression in a mouse model of muscular dystrophy. Science. 2016;351:400-3.

33. Long C, MCAnally JR, Shelton JM, Mireault AA, Bassel-Duby R, Olson EN. Prevention of muscular dystrophy in mice by CRISPR/Cas9-mediated editing of germline DNA. Science. 2014;345:1184-8.

34. Nelson CE, Hakim CH, Ousterout DG, Thakore PI, Moreb EA, Castellanos Rivera RM, Madhavan S, Pan X, Ran FA, Yan WX, et al. In vivo genome editing improves muscle function in a mouse model of Duchenne muscular dystrophy. Science. 2016;351:403-7.

35. Tabebordbar M, Zhu K, Cheng JK, Chew WL, Widrick JJ, Yan WX, Maesner C, Wu EY, Xiao R, Ran FA, et al. In vivo gene editing in dystrophic mouse muscle and muscle stem cells. Science. 2016;351:407-11.

36. Wu Y, Liang D, Wang Y, Bai M, Tang W, Bao S, Yan Z, Li D, Li J. Correction of a genetic disease in mouse via use of CRISPR-Cas9. Cell Stem Cell. 2013;13: 659-62.

37. Pellman D. Cell biology: aneuploidy and cancer. Nature. 2007;446:38-9.

38. Williams BR, Prabhu VR, Hunter KE, Glazier CM, Whittaker CA, Housman DE, Amon A. Aneuploidy affects proliferation and spontaneous immortalization in mammalian cells. Science. 2008;322:703-9.

39. Sheltzer JM, Blank HM, Pfau SJ, Tange Y, George BM, Humpton TJ, Brito IL, Hiraoka Y, Niwa O, Amon A. Aneuploidy drives genomic instability in yeast. Science. 2011;333:1026-30.

40. Tang YC, Williams BR, Siegel JJ, Amon A. Identification of aneuploidyselective antiproliferation compounds. Cell. 2011;144:499-512.
41. Cong L, Ran FA, Cox D, Lin S, Barretto R, Habib N, Hsu PD, Wu X, Jiang W, Marraffini LA, Zhang F. Multiplex genome engineering using CRISPR/Cas systems. Science. 2013;339:819-23.

42. Heidenreich M, Zhang F. Applications of CRISPR-Cas systems in neuroscience. Nat Rev Neurosci. 2016;17:36-44.

43. Mali P, Yang L, Esvelt KM, Aach J, Guell M, DiCarlo JE, Norville JE, Church GM. RNA-guided human genome engineering via Cas9. Science. 2013;339: 823-6.

44. Yang Y, Liu B, Xu J, Wang J, Wu J, Shi C, Xu Y, Dong J, Wang C, Lai W, et al. Derivation of pluripotent stem cells with in vivo embryonic and extraembryonic potency. Cell. 2017;169:243-57. e225.

45. Chiang C, Layer RM, Faust GG, Lindberg MR, Rose DB, Garrison EP, Marth GT, Quinlan AR, Hall IM. SpeedSeq: ultra-fast personal genome analysis and interpretation. Nat Methods. 2015;12:966-8.

46. Garrison E, Marth G. Haplotype-based variant detection from short-read sequencing. arXiv 2016:1207.3907

47. Bae S, Park J, Kim JS. Cas-OFFinder: a fast and versatile algorithm that searches for potential off-target sites of Cas9 RNA-guided endonucleases. Bioinformatics. 2014;30:1473-5.

48. Frock RL, Hu J, Meyers RM, Ho YJ, Kii E, Alt FW. Genome-wide detection of DNA double-stranded breaks induced by engineered nucleases. Nat Biotechnol. 2015:33:179-86.

49. Saito T, Nakatsuji N. Efficient gene transfer into the embryonic mouse brain using in vivo electroporation. Dev Biol. 2001;240:237-46.

\section{Submit your next manuscript to BioMed Central and we will help you at every step:}

- We accept pre-submission inquiries

- Our selector tool helps you to find the most relevant journal

- We provide round the clock customer support

- Convenient online submission

- Thorough peer review

- Inclusion in PubMed and all major indexing services

- Maximum visibility for your research

Submit your manuscript at www.biomedcentral.com/submit 